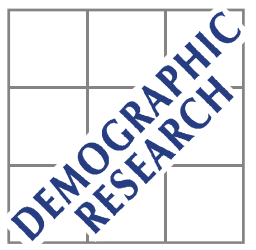

Demographic Research a free, expedited, online journal of peer-reviewed research and commentary in the population sciences published by the Max Planck Institute for Demographic Research Konrad-Zuse Str. 1, D-18057 Rostock · GERMANY www.demographic-research.org

DEMOGRAPHIC RESEARCH

VOLUME 13, ARTICLE 11, PAGES 231-280

PUBLISHED 17 NOVEMBER 2005

http://www.demographic-research.org/Volumes/Vol13/11/

DOI: 10.4054/DemRes.2005.13.11

Research Article

\title{
On the relationship between period and cohort mortality
}

\section{John R. Wilmoth}

This article is part of Demographic Research Special Collection 4, "Human Mortality over Age, Time, Sex, and Place: The $1^{\text {st }}$ HMD Symposium".

Please see Volume 13, publications 13-10 through 13-20.

(C) 2005 Max-Planck-Gesellschaft. 


\section{Table of Contents}

1 Introduction $\quad 232$

$2 \quad$ Overview and fundamental concepts 233

$\begin{array}{lll}2.1 & \text { Cohorts vs. periods } & 234\end{array}$

2.2 Quantum vs. tempo 234

$2.3 \quad$ Population dynamics vs. synthetic cohorts 234

$2.4 \quad$ Causes and consequences of partial (or surplus) quantum 236

$2.5 \quad$ Models of mortality change over time 238

$3 \quad$ Mortality functions and basic relationships 238

$3.1 \quad$ Single cohort model 238

$3.2 \quad$ Standard period-cohort model 240

$3.3 \quad$ Cohort distributions of age at death 242

$4 \quad$ Alternative measures of period mean lifespan 248

$4.1 \quad$ Relative size of a constant-birth population, $e_{0}^{*} \quad 249$

4.2 Mean age at death in a constant-birth population, $e_{0}^{\prime} \quad 249$

4.3 Tempo-adjusted life expectancy at birth, $e_{0}^{B F}$ or $e_{0}^{*}$

4.4 Comparison to period life expectancy at birth, $e_{0}$

$5 \quad$ Trends in life expectancy at birth by period and cohort 255

$5.1 \quad$ Speed of change in historical trends 255

5.2 Intrinsic difference in period-cohort trends 257

5.3 Period-cohort trends in the linear shift model 258

$\begin{array}{lll}5.4 & \text { Empirical application of the linear shift model } & 261\end{array}$

6 Comparison of six measures of mean lifespan 265

$\begin{array}{lll}7 & \text { Conclusion } & 267\end{array}$

8 Acknowledgements $\quad 268$

$\begin{array}{ll}\text { References } & 269\end{array}$

$\begin{array}{ll}\text { Appendix } & 271\end{array}$

A-1. Percentile slopes of cohort distributions of age at death 271

A-2. Fundamental properties of the linear shift model 272 


\title{
On the relationship between period and cohort mortality
}

\author{
John R. Wilmoth ${ }^{1}$
}

\begin{abstract}
In this paper I explore the formal relationship between period and cohort mortality, focusing on a comparison of measures of mean lifespan. I consider not only the usual measures (life expectancy at birth for time periods and birth cohorts) but also some alternative measures that have been proposed recently.

I examine (and reject) the claim made by Bongaarts and Feeney that the level of period $e_{0}$ is distorted, or biased, due to changes in the timing of mortality. I show that their proposed alternative measure, called "tempo-adjusted" life expectancy, is exactly equivalent in its generalized form to a measure proposed by both Brouard and Guillot, the cross-sectional average length of life (or $C A L$ ), which substitutes cohort survival probabilities for their period counterparts in the calculation of mean lifespan. I conclude that this measure does not in any sense correct for a distortion in period life expectancy at birth, but rather offers an alternative measure of mean lifespan that is approximately equal to two analytically interesting quantities: 1) the mean age at death in a given year for a hypothetical population subject to observed historical mortality conditions but with a constant annual number of births; and 2) the mean age at death, $\lambda$, for a cohort born $\lambda$ years ago.

However, I also observe that the trend in period $e_{0}$ does indeed offer a biased depiction of the pace of change in mean lifespan from cohort to cohort. Holding other factors constant, an historical increase in life expectancy at birth is somewhat faster when viewed from the perspective of cohorts (i.e., year of birth) than from the perspective of periods (i.e., year of death).
\end{abstract}

This article is part of Demographic Research Special Collection 4, "Human Mortality over Age, Time, Sex, and Place: The $1^{\text {st }}$ HMD Symposium". Please see Volume 13, Publications 13-10 through 13-20. 


\section{Introduction}

A classic problem in formal demography is how to define summary measures of demographic events for time periods that correspond in some meaningful way to the lived experience of actual cohorts. Although such period measures may not be equivalent to the analogous measure for any particular cohort, they should nevertheless represent the lifetime experience of a hypothetical cohort that is subject throughout its life to currently observed demographic conditions. The question, of course, is how to define the concept of current conditions, especially when such conditions are changing. For example, several authors have pointed out that in some situations the standard measure of lifetime completed fertility, the total fertility rate (TFR), misrepresents the average number of births that a woman would bear over her lifetime (Hajnal, 1947; Ryder, 1964; Bongaarts and Feeney, 1998). Since the problem is caused by changes from year to year in the timing of fertility as a function of age, this phenomenon is now commonly referred to as "tempo distortion," or "tempo bias."

In the case of fertility, the existence of such a distortion is widely acknowledged, even though there are differences of opinion about how best to adjust the TFR to remove such bias (Schoen, 2004). In the case of mortality, however, the recent claim by Bongaarts and Feeney $(2002,2003)$ of a similar bias affecting period life expectancy at birth, $e_{0}$, has not found wide acceptance. Without doubt, such skepticism derives in part from the dissimilarity of the two examples, since the TFR measures the number of births over the life course, whereas $e_{0}$ depicts the average age at death. This difference recalls Ryder's emphasis on the fundamental distinction between the quantum and the tempo of demographic events (Ryder, 1978).

The recent discussion of these topics has revealed a pressing need to clarify the meaning of various summary measures of average longevity in a population. Therefore, in this paper I explore the formal relationship between period and cohort mortality, with a particular emphasis on the concept of mean lifespan. I consider not only the usual measures (life expectancy at birth for periods and cohorts) but also some alternative measures that have been proposed recently.

I examine (and reject) the assertion that the level of period $e_{0}$ is distorted, or biased, due to changes in the timing of mortality. I show that the alternative measure proposed by Bongaarts and Feeney, called "tempo-adjusted" life expectancy, is exactly equivalent in its generalized form to a measure proposed by both Brouard (1986) and Guillot (2003), known as the cross-sectional average length of life (or $C A L$ ), which substitutes cohort probabilities of survival for their period counterparts in the calculation of mean lifespan. I conclude that this measure does not in any sense correct for a distortion in period life expectancy at birth, but rather offers an alternative 
measure of mean lifespan that is approximately equal to two analytically interesting quantities: 1) the mean age at death in a given year for a hypothetical population subject to observed historical mortality conditions but with a constant annual number of births; and 2) the mean age at death, $\lambda$, for a cohort born $\lambda$ years ago.

However, I also observe that the trend in period $e_{0}$ does indeed offer a biased depiction of the pace of change in mean lifespan from cohort to cohort. Holding other factors constant, an historical increase in life expectancy at birth is somewhat faster when viewed from the perspective of cohorts (i.e., year of birth) than from the perspective of periods (i.e., year of death).

The analysis begins in Section 2 with a verbal discussion of some key topics. This is followed by Section 3, which defines various mathematical functions and derives the standard period-cohort model of mortality. Mathematically sophisticated readers may wish to begin with Section 3. Likewise, individuals who are already well-versed in the specific topics addressed in this paper may wish to skip immediately to Section 3.3, or even Section 4.

\section{Overview and fundamental concepts}

Demographic events mark major life course transitions (e.g., birth, marriage, fertility, migration, retirement, widowhood, death). Their likelihood of occurrence within some time interval is often described using rates (and/or conditional probabilities), whose specificity may vary as a function of age, time, sex, and other individual characteristics. Such rates are often used to calculate a variety of summary measures that depict the intensity and/or timing of such events over the life course. Undoubtedly, the two most common of these measures are life expectancy at birth, $e_{0}$, and the total fertility rate (TFR).

An overview of demographic summary measures must begin with certain fundamental concepts, including three important dichotomies: (a) cohorts vs. periods; (b) quantum vs. tempo; and (c) population dynamics vs. synthetic cohorts. In addition to these three distinctions, we need to understand the phenomenon of partial (or surplus) quantum, which affects the period TFR (and all measures of quantum) whenever the timing of fertility (or other event) is changing over time. To address these and other issues in this paper, I describe a new class of models that can be used to explore mortality (and other demographic) trends based on assumptions about changes in the age distribution of events, rather than the age pattern of risk. 


\subsection{Cohorts vs. periods}

Cohorts and periods are two different ways of reckoning time when analyzing demographic events. A cohort is an actual group of persons who experience a major life event around the same time. For example, birth cohorts are composed of individuals who are born in the same year (or decade, etc.). Cohort life expectancy at birth is the observed average age at death for this group (ignoring migration). In the same context, a period is a time interval (e.g., year, decade) and is associated with a synthetic cohort, which is an imaginary group of people who experience, hypothetically, the demographic conditions of that period throughout life. Thus, period life expectancy at birth is the expected average age at death for a synthetic cohort that experiences the mortality risks of that time (as reflected in age-specific death rates) from birth onward.

\subsection{Quantum vs. tempo}

In general, quantum refers to the intensity (or level, or frequency) with which some demographic event occurs in a population. Quantum can be described as a function of age (e.g., age-specific rates) or summarized over the entire life course (e.g., the lifetime count or probability of an event). Age-specific measures of quantum always have the number of events in the numerator. In the case of mortality, these include death counts, probabilities of death or survival, and death rates. In contrast, tempo refers to the timing of a demographic event over the life course. Measures of tempo are expressed in units of time (or age) and usually depict the duration until an event's occurrence. The most common example is life expectancy at birth, but other measures of mortality tempo include percentiles of the distribution of age at death (e.g., median age at death) and person-years of survival (within some interval of time and/or age).

\subsection{Population dynamics vs. synthetic cohorts}

There are two classes of period measures used for summarizing the demographic events of a given time interval: (a) those that describe population dynamics, and (b) those that depict the hypothetical experience of a synthetic cohort. These two types of measures serve different purposes, and a measure that is appropriate in one case may be inappropriate in the other. For example, the period total fertility rate (TFR), which equals the sum of observed age-specific fertility rates for a given period, depicts accurately the average contribution to population change attributable to the current 
fertility of women in the reproductive age range (Calot, 2001). From this perspective, the TFR is a useful and reliable measure of population dynamics.

However, as a measure of lifetime fertility for a synthetic cohort, the TFR has at least two inherent flaws. First, as discussed in the following section, it is affected by the phenomenon of partial (or surplus) quantum whenever there are changes in the timing of fertility as a function of age. This problem, often called "tempo distortion" or "tempo bias," can be circumvented by a small adjustment applied to age-specific fertility rates, which has the effect of replacing (or removing) the partial (or surplus) quantum caused by changes in fertility timing. Second, observed age-specific fertility rates reflect past as well as current fertility patterns, since they depend on the distribution of women by parity at each age. This latter problem could be avoided by computing an alternative measure of period total fertility based on parity transition rates within a multi-state framework.

Thus, even though it is usually presented as a measure of lifetime fertility for a synthetic cohort, it is more appropriate to interpret the TFR as a measure of population dynamics. $^{2}$ If we desire a measure of total fertility that depicts the lifetime experience of a synthetic cohort based only on current fertility conditions, then we must address both of the problems mentioned above. Perhaps the ideal solution would consist of replacing the traditional TFR by a pair of period measures: (a) the net reproduction rate $(N R R)$ for the analysis of population dynamics, and (b) a full-quantum (or tempoadjusted) multi-state $T F R$ to represent the lifetime reproduction of a synthetic cohort.

In the case of mortality as well, some measures of mean lifespan are useful mostly for the analysis of population dynamics. For example, the cross-sectional average length of life $\left(C A L\right.$, or $\left.e_{0}^{*}\right)$ depicts the relative size of a population at a point in time given its past mortality trends but assuming (hypothetically) a constant annual stream of births (Guillot, 2003). As shown here, CAL is also approximately equal to certain measures of mean lifespan for the population in question. For example, it is quite similar in form to $e_{0}^{\prime}$, defined to be the mean age at death $(M A D)$ that would be observed in a given time period for a population with an identical historical mortality pattern and a constant annual number of births (Bongaarts and Feeney, 2003). However, such measures describe population dynamics, not the life course of a synthetic cohort based exclusively on the mortality risks of a given period. I show here that both $e_{0}^{*}$ and $e_{0}^{\prime}$ depend on past as well as present death rates; in contrast, the period life expectancy at birth, $e_{0}$, is the expected mean age at death implied by the observed death rates of that time alone.

\footnotetext{
${ }^{2}$ The $T F R$ is often interpreted (at least implicitly) as a proxy for the net reproduction rate (NRR). For example, since population replacement in low-mortality populations requires a $T F R$ of about 2.1 children per woman, a convenient approximation in such situations is $N R R \approx T F R / 2.1$.
} 
In general, period measures of the average age of some life course event (e.g., death) at time $t$ have two common forms: (a) the mean age of the event that is or would be observed in the population at that time, perhaps under some set of hypothetical conditions (e.g., assuming a constant stream of births over time); or (b) the expected mean age of the event in a synthetic cohort assuming that current age-specific transition rates are experienced over a lifetime. Some confusion results from the fact that different traditions have existed in fertility and mortality analysis concerning the appropriate definition for the period mean age of the event. Perhaps because a central focus of fertility studies has been the role of reproduction in population dynamics, the definition of "average age at birth" has followed the concept of an observed mean age. In contrast, it was quite sensible for life expectancy at birth to reflect the concept of an expected mean age, since mortality studies have been framed in terms of risk reduction and abstract notions of quality of life, not population dynamics.

\subsection{Causes and consequences of partial (or surplus) quantum}

Many demographic events, like death, occur at various ages for members of the same cohort. An associated probability distribution depicts the timing of such events as a function of age, and thus also in relation to the time periods in which they occur. During a given time period, each living cohort undergoes some fraction of its total lifetime experience of the event in question, and the total number of events observed during that period is a composite of these fractional segments of cohort lifetimes.

If the age distribution of events is identical from cohort to cohort, a period crosssection of these fractional segments sums to one, and therefore the collection of events within the period can be said to represent the equivalent of one complete cohort lifetime. However, whenever there are changes in the distribution of events by age for successive cohorts, a period cross-section of cohort probability distributions typically does not sum to one. A delay in the timing of events from cohort to cohort produces a phenomenon of partial quantum, whereas an acceleration of timing results in surplus quantum during the period in question. In such cases, events observed during a given period generally misrepresent the equivalent of one complete cohort lifetime. ${ }^{3}$ (To simplify the exposition here, I will often consider only the case of tempo delay and partial quantum, since the causes and consequences of surplus quantum are identical, though always in the opposite direction.)

\footnotetext{
${ }^{3}$ A sum of one in this case could occur only by coincidence, if negative and positive factors cancelled out, but such an occurrence seems extremely unlikely.
} 
The phenomenon of partial (or surplus) quantum is the source of a tempo distortion, or bias, that affects measures of lifetime quantum, like the TFR. This distortion can be easily eliminated by adjusting age-specific fertility rates in an appropriate fashion. However, as noted earlier, this distortion is relevant only in situations where the TFR is interpreted as a measure of lifetime fertility for a synthetic cohort. When the TFR is employed as a measure of population dynamics, the partial (or surplus) quantum caused by changes in fertility tempo is a desirable outcome. In such cases adjusting the measure to remove tempo effects creates a bias where none existed before.

The role of these factors in the analysis of quantum measures, like the $T F R$, is relatively straightforward, owing to the fact that the model of a synthetic cohort is relatively simple in this case. In order to represent the lifetime quantum of an event, such as total fertility, demographers have typically created a synthetic cohort that is not subject to mortality or other forms of attrition, and thus the base population that accumulates events (e.g., births) over the life course is constant. For this reason, adjusting for the effects of partial quantum (or tempo delay) is a simple matter of replacing the fraction of events for each cohort that have been postponed from the time period in question into the future.

In contrast, tempo measures and their associated synthetic cohorts have a more complicated mathematical structure due to the phenomenon of attrition, which affects the base population (e.g., number of survivors) that is eligible to experience a given event (e.g., death). In such cases, adjusting for tempo delay (or partial quantum) has a dual effect. For a given base population, it restores a fraction of events that have been postponed into the future. However, it also alters the base population itself at each age. Whereas the first effect has a relatively minor impact on measures of mean age (e.g., life expectancy at birth), the latter effect is quite significant and fundamentally alters the nature of the measure. In fact, as I show here, tempo adjustment has the effect of converting a period survival probability (i.e., the probability of survival to age $x$ within a period life table) into an analogous cohort survival probability (i.e., the probability of survival to age $x$ for the cohort born $x$ years ago). In doing so, it converts period $e_{0}$ into $C A L$, and thus fundamentally alters the nature of the measure (recall the earlier discussion of synthetic cohorts vs. population dynamics).

In short, adjusting for tempo change in the case of a tempo measure has the effect of removing historical changes in the quantity being measured. Tempo adjustment in this case converts a period measure based on a synthetic cohort into a cross-sectional measure that reflects the past experiences of cohorts. As noted earlier, the primary use for $C A L$ is the analysis of population dynamics. Differences between $C A L$ and period $e_{0}$ do not suggest that the latter measure is "distorted" in any sense. Rather, the two measures differ because they describe different things. 


\subsection{Models of mortality change over time}

This analysis uses a relatively new class of models to gain insights into period-cohort relationships. Previously, most models of mortality change over age and time have been specified as a function of trends in age-specific death rates. Here, following the example of Bongaarts and Feeney $(2002,2003)$, changes in mortality are specified in terms of shifting distributions of deaths by age. The former type might be referred to as "rate models," whereas the latter could be called "percentile models."

\section{Mortality functions and basic relationships}

\subsection{Single cohort model}

For a single cohort (real or synthetic), the usual formulas for computing life expectancy at birth are the following:

$$
\begin{aligned}
e_{0} & =\int_{0}^{\infty} x \phi(x) d x \\
& =\int_{0}^{\infty} x \ell(x) \mu(x) d x \\
& =\int_{0}^{\infty} \ell(x) d x,
\end{aligned}
$$

where $\phi(x)=-\frac{d}{d x} \ell(x)=\ell(x) \mu(x)$ is the probability density function, describing the distribution of deaths by age in the cohort; $\mu(x)=-\frac{d}{d x} \ell(x) / \ell(x)=-\frac{d}{d x} \ln \ell(x)$ is the death rate at age $x ; H(x)=\int_{0}^{x} \mu(a) d a$ is the cumulative death rate up until age $x$; and $\ell(x)=e^{-H(x)}=e^{-\int_{0}^{x} \mu(a) d a}=\int_{x}^{\infty} \phi(a) d a$ is the probability of survival from birth until exact age $x$.

Although they have different forms, all three formulas in equation 1 yield the same value for the mean age at death in a cohort. The difference between the first two formulas is trivial, since $\phi(x)=\ell(x) \mu(x)$. Both of these formulas depict life expectancy at birth as an average age at death, or as an expected value associated with the probability distribution. However, the last formula is different in both form and substance; it suggests an alternative interpretation of mean lifespan as the accumulation of person-years lived, on average, by members of the cohort. 
It is also possible to depict life expectancy at birth as an integral with respect to the (unconditional) probability of dying, rather than age. Such calculations are closely related to percentiles of the distribution, $\tilde{a}(\pi)$, which are defined as follows:

$$
\tilde{a}(\pi)=x \text { such that } \pi=\Phi(x)=1-\ell(x),
$$

where $\Phi(x)=\int_{0}^{x} \phi(a) d a$ is the distribution (or cumulative probability) function for ages at death in the cohort. Thus, $\tilde{a}(\pi)$ is an age, $x$, such that the proportion of total deaths (over the cohort's lifetime) occurring before age $x$ is $\pi$. The derivative of $\pi$ with respect to age, $x$, equals the probability density function at that age:

$$
\frac{d}{d x} \pi=\phi(x)
$$

Substituting $\tilde{a}(\pi)$ in place of $x$, the relationships described in equations 2 and 3 can also be written as follows:

$$
\pi=\Phi(\tilde{a}(\pi)) \quad \text { and } \quad \frac{d}{d x} \pi=\phi(\tilde{a}(\pi))
$$

Moreover, substituting $x=\tilde{a}(\pi)$ and $d \pi=\phi(x) d x$ in equation 1, and recalling that $\ell(x) / \phi(x)=\mu(x)$, we obtain the following alternative forms for life expectancy at birth:

$$
\begin{aligned}
e_{0} & =\int_{0}^{1} \tilde{a}(\pi) d \pi \\
& =\int_{0}^{1} \frac{1}{\mu(\tilde{a}(\pi))} d \pi .
\end{aligned}
$$

Thus, if we assign equal weight to arbitrarily small intervals of age, each containing an equal share of the lifetime probability of death (totaling one, of course), then life expectancy at birth equals the average of either the midpoint of each age interval or the reciprocal of the death rate within each interval. 


\subsection{Standard period-cohort model}

The above formulas describe the calculation of life expectancy at birth for just one cohort, which could be either an actual birth cohort or a synthetic cohort derived from the collective mortality experience of cohorts alive during some time period. Using long series of historical data (mostly from vital statistics and census data), a common problem is to construct series of annual life tables for both periods and cohorts (e.g., the Human Mortality Database, www.mortality.org). To accomplish this goal, it is necessary to make some assumption about the link between period and cohort mortality, so that the two sets of tables are related in some logical and consistent manner.

The traditional manner of defining this link has been to equate period and cohort mortality in terms of their age-specific death rates. Thus, we typically begin by assuming that the death rates for a period life table should be derived directly from observed cohort death rates. In continuous age and time, this relationship can be expressed as follows:

$$
\mu(x, t)=\mu_{p}(x, t)=\mu_{c}(x, t-x)=\mu_{c}(x, \tau)
$$

where $\tau=t-x$. Thus, by definition, the period death rate at age $x$ and time $t$, $\mu(x, t)=\mu_{p}(x, t)$, equals $\mu_{c}(x, t-x)=\mu_{c}(x, \tau)$, the death rate at age $x$ for the cohort born $x$ years ago at time $\tau$. Given this assumption, a series of historical life tables for both periods and cohorts is fully defined by the surface of age-specific rates expressed as a function of age and time. ${ }^{4}$

For example, life expectancy at birth for a given period $t$ can be computed using the above equations. Written using a complete notation, the standard equations for period life expectancy at birth are as follows:

\footnotetext{
${ }^{4}$ The equations given here refer to the death rate at a point of age and time, $(x, t)$, which simplifies the task of defining the link between period and cohort mortality. In practice, period and cohort mortality must be defined and measured over some time interval, such as a single calendar year. In such situations, one simple approach is merely to equate period rates to cohort rates, or vice versa, without further manipulation. However, the rates that result from such a procedure are less precise in terms of their temporal specificity than what is obtained by constructing different sets of overlapping rates for periods and cohorts. Although derived from the same data, accurate mortality rates for periods and cohorts over discrete intervals are estimated by altering slightly the configuration of age and time used to organize the raw data (annual death counts, and estimates of exposure-to-risk in person-years), so that each set of rates corresponds to exact period or cohort age intervals. For purposes of the present discussion, such complications can safely be ignored, since the mathematical development pursued here is expressed entirely in terms of continuous age and time.
} 


$$
\begin{aligned}
e_{0}^{p}(t) & =\int_{0}^{\infty} x \phi_{p}(x, t) d x \\
& =\int_{0}^{\infty} x \ell_{p}(x, t) \mu_{p}(x, t) d x \\
& =\int_{0}^{\infty} \ell_{p}(x, t) d x,
\end{aligned}
$$

where $\phi_{p}(x, t)=-\frac{d}{d x} \ell_{p}(x, t)=\ell_{p}(x, t) \mu_{p}(x, t)$ gives the probability distribution of ages at death for the synthetic cohort of period $t ; \mu_{p}(x, t)=\mu(x, t)=-\frac{d}{d x} \ell_{p}(x, t) / \ell_{p}(x, t)$ $=-\frac{d}{d x} \ln \ell_{p}(x, t)$ is the death rate at age $x$ and time $t ; H_{p}(x, t)=\int_{0}^{x} \mu_{p}(a, t) d a$ is the cumulative rate function at age $x$ and time $t$; and $\ell_{p}(x, t)=e^{-H_{p}(x, t)}=e^{-\int_{0}^{x} \mu_{p}(a, t) d a}$ $=\int_{x}^{\infty} \phi_{p}(a, t) d a$ is the period probability of survival from birth until exact age $x$.

Similarly, life expectancy at birth for a cohort born at time $\tau$ can be computed as follows:

$$
\begin{aligned}
e_{0}^{c}(\tau) & =\int_{0}^{\infty} x \phi_{c}(x, \tau) d x \\
& =\int_{0}^{\infty} x \ell_{c}(x, \tau) \mu_{c}(x, \tau) d x \\
& =\int_{0}^{\infty} \ell_{c}(x, \tau) d x,
\end{aligned}
$$

where $\phi_{c}(x, \tau)=-\frac{d}{d x} \ell_{c}(x, \tau)=\ell_{c}(x, \tau) \mu_{c}(x, \tau)$ gives the probability distribution of ages at death for the cohort born at time $\tau ; \mu_{c}(x, \tau)=\mu(x, \tau+x)=-\frac{d}{d x} \ell_{c}(x, \tau) / \ell_{c}(x, \tau)$ $=-\frac{d}{d x} \ln \ell_{c}(x, \tau)$ is the cohort death rate at age $x ; H_{c}(x, \tau)=\int_{0}^{x} \mu_{c}(a, \tau) d a$ is the cumulative rate function at age $x$; and $\ell_{c}(x, \tau)=e^{-H_{c}(x, \tau)}=e^{-\int_{0}^{x} \mu_{c}(a, \tau) d a}=\int_{x}^{\infty} \phi_{c}(a, \tau) d a$ is the cohort probability of survival from birth until exact age $x$. 


\subsection{Cohort distributions of age at death}

Let us also define percentiles of the distribution of age at death for each cohort as follows:

$$
\tilde{a}_{c}(\pi, \tau)=x \quad \text { such that } \quad \pi=\Phi_{c}(x, \tau)=1-\ell_{c}(x, \tau),
$$

where $\Phi_{c}(x, \tau)=\int_{0}^{x} \phi_{c}(a, \tau) d a$ is the distribution (or cumulative probability) function for age at death in the cohort born at time $\tau$. An important quantity in this discussion will be speed of change in these percentiles over time. Define $s_{c}(x, \tau)$ to be the pace of change (from cohort to cohort) in the percentile of ages at death observed at age $x$ for the cohort born at time $\tau$. Thus, by definition

$$
s_{c}(x, \tau)=\frac{d}{d \tau} \tilde{a}_{c}(\pi, \tau)
$$

where $\pi=\Phi_{c}(x, \tau)$ is fixed.

In general, such quantities, known as "cohort percentile slopes," are useful for describing the relationship between period and cohort mortality. It is shown in the Appendix (see section A-1) that a cohort percentile slope has the following relationship to the other mortality functions described above:

$$
s_{c}(x, \tau)=\frac{-\frac{d}{d \tau} \Phi_{c}(x, \tau)}{\phi_{c}(x, \tau)}=\frac{\frac{d}{d \tau} \ell_{c}(x, \tau)}{\phi_{c}(x, \tau)}=\frac{\frac{d}{d \tau} \ln \ell_{c}(x, \tau)}{\mu_{c}(x, \tau)}=\frac{-\frac{d}{d \tau} H_{c}(x, \tau)}{\mu_{c}(x, \tau)} .
$$

Thus, the cohort percentile slope at age $x$ equals the ratio (either positive or negative) of the change over time in some measure of cumulative mortality or survival, divided by an associated measure of age-specific mortality.

Using the first relationship of equation 11, it is possible to derive simple expressions for the derivatives of $\Phi_{c}(x, \tau)$ in three directions:

$$
\begin{array}{ll}
\frac{d}{d \tau} \Phi_{c}(x, \tau)=-s_{c}(x, \tau) \phi_{c}(x, \tau) & \text { (horizontal) } \\
\frac{d}{d x} \Phi_{c}(x, \tau)=\phi_{c}(x, \tau) & \text { (diagonal) } \\
\frac{d}{d x} \Phi_{c}(x, t-x)=\left(1+s_{c}(x, t-x)\right) \phi_{c}(x, t-x) & \text { (vertical) }
\end{array}
$$


As illustrated here in Figure 1, the labels, "horizontal," "diagonal," and "vertical," refer to directions of change in a Lexis diagram, drawn such that the abscissa and ordinate of the Cartesian plane correspond to the time and age of death, respectively (thus, cohort lifetimes are represented by diagonal lines). The horizontal and diagonal derivatives are obtained, respectively, from the earlier equation for the percentile slope and from the definition of $\Phi_{c}(x, \tau)$ in terms of $\phi_{c}(x, \tau)$. The vertical derivative follows from the fact that the derivative in the diagonal direction equals the sum of the other two derivatives.

Figure 1: Schematic representation of derivatives in three directions of the cohort cumulative probability function, $\Phi_{c}(x, \tau)$

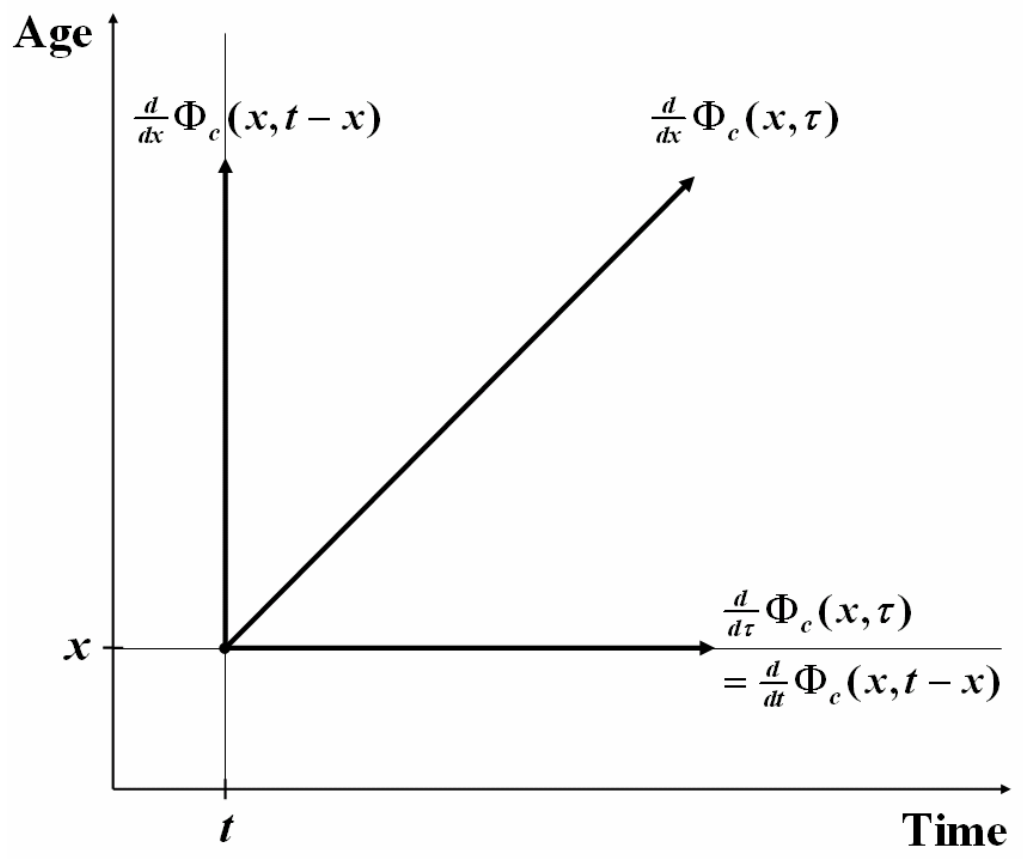

Note: By assumption, $\tau=t-x$. 
Wilmoth: On the relationship between period and cohort mortality

The derivative of $\Phi_{c}(x, \tau)$ in the vertical direction is important because it illustrates that the cross-sectional sum of cohort probability distributions does not in general equal one. For example, if $s_{c}(x, t-x)>0$ for all $x$, it follows that

$$
\begin{aligned}
\int_{0}^{\infty} \phi_{c}(x, t-x) d x & <\int_{0}^{\infty}\left(1+s_{c}(x, t-x)\right) \phi_{c}(x, t-x) d x \\
& =\int_{0}^{\infty} \frac{d}{d x} \Phi_{c}(x, t-x) d x=1 .
\end{aligned}
$$

In this example, since cohort percentile slopes at time $t$ are positive, the timing of death is being delayed or postponed for each successive cohort. This equation illustrates the phenomenon of partial quantum, which occurs whenever the age distribution of events (deaths) is shifting upward over time. Conversely, if the distribution of deaths is shifting uniformly toward younger ages (thus, the timing of death is being advanced or accelerated), then $s_{c}(x, t-x)$ would be negative for all $x$ at time $t$, and the above sum would be greater than unity (i.e., surplus quantum). Let us refer to $\phi_{c}(x, t-x)$ as a cross-sectional cohort probability density function.

Assuming that $s_{c}(x, t-x)>-1$ for all $x$ at time $t,{ }^{5}$ it is possible to define the following probability density function:

$$
\phi^{*}(x, t)=\left(1+s_{c}(x, t-x)\right) \phi_{c}(x, t-x)=\frac{\phi_{c}(x, t-x)}{1-r_{c}(x, t-x)},
$$

where $r_{c}(x, t-x)=\frac{s_{c}(x, t-x)}{1+s_{c}(x, t-x)}$, and thus $1+s_{c}(x, t-x)=\left(1-r_{c}(x, t-x)\right)^{-1}$. This function sums to one over the full age range (see equation 15 above), since $s_{c}(x, t-x) \phi_{c}(x, t-x)$ replaces the missing quantum at age $x$, assuming $s_{c}(x, t-x)>0{ }^{6}$ Thus, $\phi^{*}(x, t)$ is an adjusted cross-sectional cohort probability density function.

\footnotetext{
${ }^{5}$ It is theoretically possible for cohort percentiles to have slopes that are less than -1 , and their reality has been confirmed by empirical observation. For expediency, this situation will be not covered in this paper, as we assume that $s_{c}(x, t-x)>-1$. Although the formulas of this section remain correct even when cohort percentile slopes dip below -1 , the interpretation of the quantities, $\phi^{*}(x, t)$ and $\mu^{*}(x, t)$, as adjusted density functions and adjusted death rates is no longer valid.

${ }^{6}$ In this discussion we will generally consider the example of cohort percentiles that increase over time, reflecting an increase in longevity. It should be evident that a decrease over time is also possible (except at age 0 ) and is associated with opposite effects.
} 
One important feature of the adjusted function, $\phi^{*}(x, t)$, is its relationship to the cross-sectional cohort cumulative probability and survival functions, $\Phi_{c}(x, t-x)$ and $\ell_{c}(x, t-x)$, respectively. In the following equation, note that the first integral derives from the definition of $\Phi_{c}$, whereas the second integral follows from equation 14:

$$
\Phi_{c}(x, t-x)=\int_{0}^{x} \phi_{c}(a, t-x) d a=\int_{0}^{x} \phi^{*}(a, t) d a
$$

Likewise, it follows that:

$$
\ell_{c}(x, t-x)=1-\Phi_{c}(x, t-x)=\int_{x}^{\infty} \phi_{c}(a, t-x) d a=\int_{x}^{\infty} \phi^{*}(a, t) d a .
$$

These relationships, linking $\Phi_{c}$ and $\ell_{c}$ to $\phi_{c}$ and $\phi^{*}$, are illustrated here in Figure 2A. Following a similar logic, let us define adjusted death rates as follows:

$$
\mu *(x, t)=\left(1+s_{c}(x, t-x)\right) \mu(x, t)=\frac{\mu(x, t)}{1-r_{c}(x, t-x)} .
$$

The cumulative death rate at age $x$ and time $t$ also has two equivalent forms:

$$
H_{c}(x, t-x)=\int_{0}^{x} \mu_{c}(a, t-x) d a=\int_{0}^{x} \mu^{*}(a, t) d a
$$

Therefore, the cohort survival probability at age $x$ and time $t$ can be computed using either set of death rates:

$$
\ell_{c}(x, t-x)=\exp \left\{-\int_{0}^{x} \mu_{c}(a, t-x) d a\right\}=\exp \left\{-\int_{0}^{x} \mu^{*}(a, t) d a\right\} .
$$

These relationships, linking $-\ln \ell_{c}$ to $\mu$ and $\mu^{*}$, are illustrated here in Figure 2B. 
Figure 2: Illustration of relationship between cumulative quantum for cohorts and "tempo-adjusted" age-specific quantum for periods

A) Cohort cumulative probability of death, $\Phi_{c}(x, \tau)$, and probability of survival, $\ell_{c}(x, \tau)$, as functions of both $\phi_{c}(a, \tau)$ and $\phi^{*}(a, t)$

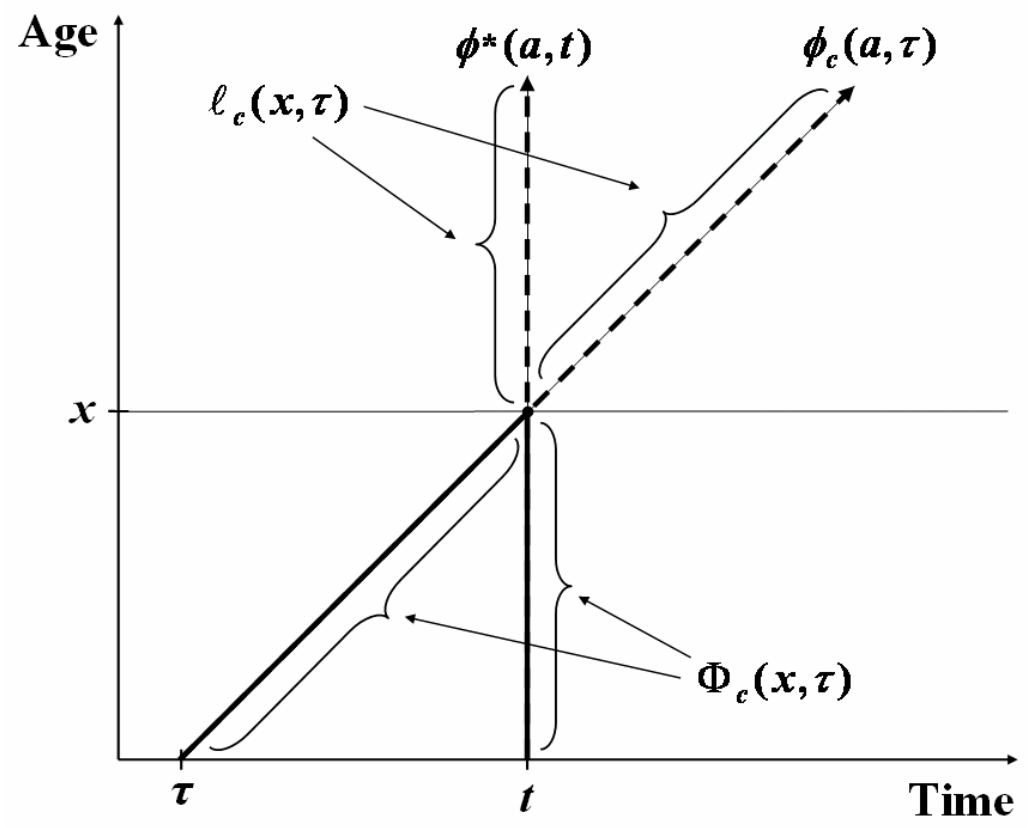

Note: As discussed in the text, $\Phi_{c}(x, \tau)=\int_{0}^{x} \phi_{c}(a, \tau) d a=\int_{0}^{x} \phi^{*}(a, t) d a$, and

$\ell_{c}(x, \tau)=1-\Phi_{c}(x, \tau)=\int_{x}^{\infty} \phi_{c}(a, \tau) d a=\int_{x}^{\infty} \phi^{*}(a, t) d a$, where $\tau=t-x$. Compare equations 17 and 18. 


\section{Figure 2: Continued}

B) Cohort probability of survival, $\ell_{c}(x, \tau)$, as functions of both $\mu_{c}(a, \tau)$ and $\mu^{*}(a, t)$

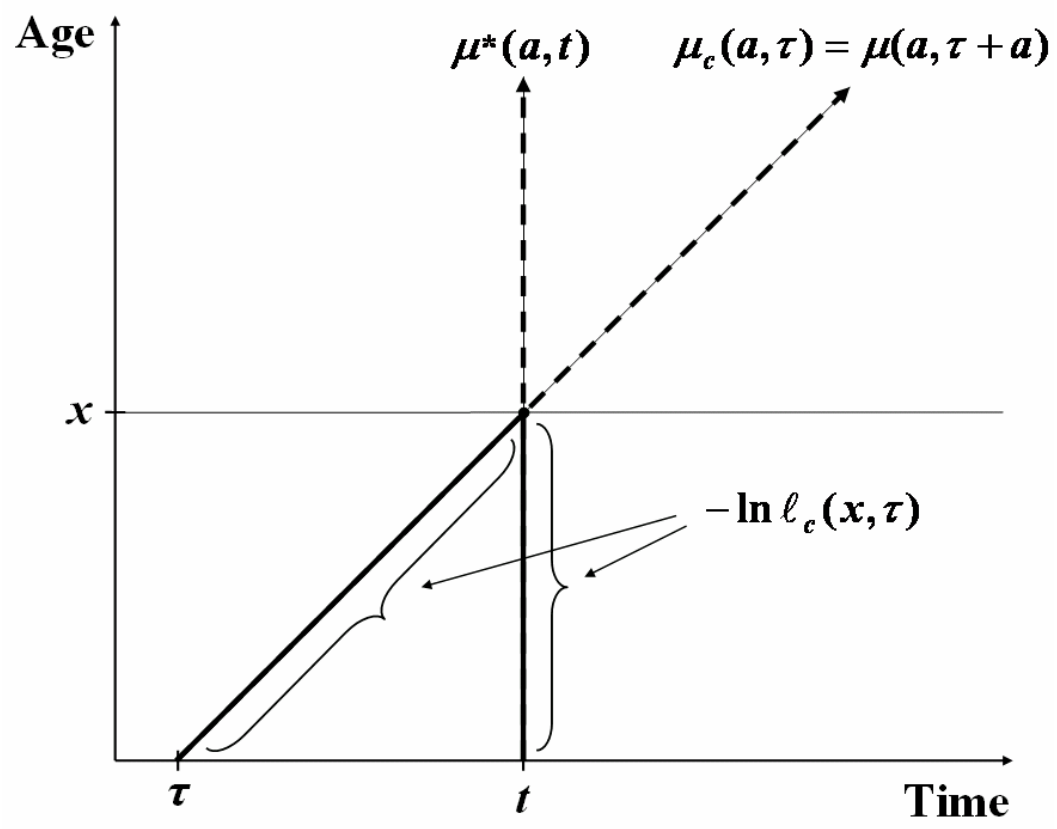

Note: As discussed in the text, $\ell_{c}(x, \tau)=\exp \left\{-\int_{0}^{x} \mu_{c}(a, \tau) d a\right\}=\exp \left\{-\int_{0}^{x} \mu^{*}(a, t) d a\right\}$, where $\tau=t-x$. Compare equation 21 . 


\section{Alternative measures of period mean lifespan}

In addition to life expectancy at birth, $e_{0}$, several other measures of period mean lifespan have recently been put forth (see review by Bongaarts, 2005). Here, we focus on two measures in particular: the "cross-sectional average length of life" $(C A L)$, proposed by Brouard (1986) and Guillot (2003), and "tempo-adjusted" life expectancy at birth, suggested by Bongaarts and Feeney $(2002,2003)$. In this section, I will explore the mathematical relationship between these and one related measures, plus their connection to period $e_{0}$.

In terms of notation, let $C A L$ be denoted $e_{0}^{*}$, and let the Bongaarts-Feeney measure be written as $e_{0}^{B F}$. In this section we will also consider a third measure, $e_{0}^{\prime}$, or the mean age at death $(M A D)$ in a constant-birth population. As we shall see, these three period measures are closely related; moreover, they are equivalent in a special case (i.e., when the shift at time $t$ in cross-sectional cohort distributions of age at death is constant

across age). However, $e_{0}^{*}, e_{0}^{\prime}$, and $e_{0}^{B F}$ are different in general from the period life expectancy at birth, $e_{0}$. All four measures are equal only under a very restrictive condition (i.e., that age-specific mortality rates are not changing at time $t$ and have been constant over time for all living cohorts).

A key contention of this article is that $e_{0}^{*}$ and $e_{0}^{\prime}$ are useful primarily as measures of population dynamics and, as such, differ fundamentally from $e_{0}$, which is based on the model of a synthetic cohort. As discussed earlier, the synthetic cohort that underlies $e_{0}$ is a hypothetical group of people who experience the death rates of the current period throughout life. In contrast, a model of population dynamics underlies the interpretation of $e_{0}^{*}$ and $e_{0}^{\prime}$; the key feature of this model is an assumption of a constant stream of births flowing into the population, arriving at rate of $B$ births per year. If this constant-birth population were subject to the historical mortality conditions of some actual population up until time $t$, then at that moment its size would be $B e_{0}^{*}(t)$, and the mean age at death observed in the population would be $e_{0}^{\prime}(t)$. Although $e_{0}^{B F}(t)$, the measure proposed by Bongaarts and Feeney, may serve as an approximation for these other two measures, it has no clear interpretation of its own except in the special case (mentioned earlier) where it equals the other two measures. 


\subsection{Relative size of a constant-birth population, $e_{0}^{*}$}

Both Brouard (1986) and Guillot (2003) have proposed the "cross-sectional average length of life," a measure known by its acronym, $C A L$, written here also as $e_{0}^{*}$. By definition,

$$
\begin{aligned}
e_{0}^{*}(t) & =C A L(t)=\int_{0}^{\infty} \ell_{c}(x, t-x) d x \\
& =\int_{0}^{\infty} \exp \left\{-\int_{0}^{x} \mu^{*}(a, t) d a\right\} d x=\int_{0}^{\infty} x \phi^{*}(x, t) d x,
\end{aligned}
$$

where $\mu^{*}(x, t)$ and $\phi^{*}(x, t)$ are defined as before. Despite the similarity of these

formulas to those underlying the calculation of life expectancy at birth, $C A L$ or $e_{0}^{*}$ is useful primarily as a measure of population dynamics. As noted by Guillot (2003), in a constant-birth population with a steady inflow of $B$ births per year, the density of survivors at exact age $x$ and time $t$ would be $B \ell_{c}(x, t-x)$. Therefore, the size of the (hypothetical) constant-birth population that would be observed at time $t$ is a simple function of $C A L(t)$ :

$$
N(t)=\int_{0}^{\infty} B \ell_{c}(x, t-x) d x=B \int_{0}^{\infty} \ell_{c}(x, t-x) d x=B e_{0}^{*}(t) .
$$

\subsection{Mean age at death in a constant-birth population, $e_{0}^{\prime}$}

By definition, $e_{0}^{\prime}(t)$ equals the mean age at death that would be observed at time $t$ given the mortality history of an actual population but assuming a constant inflow of births into the population. If there were $B$ births per year in such a population, then the density of deaths at exact age $x$ and time $t$ would be $B \phi_{c}(x, t-x)$. Therefore, the mean age at death, $M A D$, that would be observed at time $t$ in a (hypothetical) constant-birth population is as follows: 
Wilmoth: On the relationship between period and cohort mortality

$$
\begin{aligned}
e_{0}^{\prime}(t) & =\operatorname{MAD}(t)=\frac{\int_{0}^{\infty} x B \phi_{c}(x, t-x) d x}{\int_{0}^{\infty} B \phi_{c}(x, t-x) d x} \\
& =\frac{\int_{0}^{\infty} x \phi_{c}(x, t-x) d x}{\int_{0}^{\infty} \phi_{c}(x, t-x) d x}=\int_{0}^{\infty} x \frac{\phi_{c}(x, t-x)}{1-\bar{r}_{c}(t)} d x=\int_{0}^{\infty} x \phi^{\prime}(x, t) d x,
\end{aligned}
$$

where $\phi^{\prime}(x, t)=\frac{\phi_{c}(x, t-x)}{1-\bar{r}_{c}(t)}$ by definition, and where $\bar{r}_{c}(t)=\int_{0}^{\infty} r_{c}(x, t-x) \phi^{*}(x, t) d x$ is a (weighted) average of $r_{c}(x, t-x)$ for time $t$.

Equivalence of the various forms of $e_{0}^{\prime}(t)$ in equation 24 derives from the following fundamental relationship: ${ }^{7}$

$$
\int_{0}^{\infty} \phi_{c}(x, t-x) d x=\int_{0}^{\infty}\left(1-r_{c}(x, t-x)\right) \phi^{*}(x, t) d x=1-\bar{r}_{c}(t)
$$

In other words, $\bar{r}_{c}(t)$ measures the missing (or surplus) quantum at time $t$ whenever the timing of cross-sectional cohort mortality is being delayed (or accelerated) at that moment. Note that $\bar{r}_{c}(t)$ also equals the pace of change over time in $C A L(t)$ :

$$
\begin{aligned}
\frac{d}{d t} C A L(t) & =\int_{0}^{\infty} \frac{d}{d t} \ell_{c}(x, t-x) d x=\int_{0}^{\infty} \frac{s_{c}(x, t-x)}{1+s_{c}(x, t-x)} \phi^{*}(x, t) d x \\
& =\int_{0}^{\infty} r_{c}(x, t-x) \phi^{*}(x, t) d x=\bar{r}_{c}(t) .
\end{aligned}
$$

\footnotetext{
${ }^{7}$ Some authors have referred to the quantity in equation $25,1-\bar{r}_{c}(t)$, as the "total mortality rate," by analogy to the total fertility rate (Bongaarts and Feeney, 2003; Guillot, 2005). However, I have chosen not to use this terminology, because $\phi_{c}(x, t-x)$ is not a mortality rate by the usual definition of the term. Alternatively, the same concept is expressed here using the term "partial (or surplus) quantum."
} 


\subsection{Tempo-adjusted life expectancy at birth, $e_{0}^{B F}$ or $e_{0}^{*}$}

Bongaarts and Feeney $(2002,2003)$ define "tempo-adjusted" life expectancy at birth as follows:

$$
\begin{aligned}
e_{0}^{B F}(t) & =\int_{0}^{\infty} \exp \left\{-\int_{0}^{x} \frac{\mu(a, t)}{1-\bar{r}_{c}(t)} d a\right\} d x \\
& =\int_{0}^{\infty} \exp \left\{-\int_{0}^{x} \mu^{\prime}(a, t) d a\right\} d x,
\end{aligned}
$$

where $\mu^{\prime}(x, t)=\frac{\mu(x, t)}{1-\bar{r}_{c}(t)}=\frac{\mu_{c}(x, t-x)}{1-\bar{r}_{c}(t)}$ by definition. Comparing this formula to the one for $e_{0}^{\prime}(t)$ given above (i.e., equation 24), we see that each equation resembles one of the classic formulas for life expectancy at birth. It is also useful to compare $\mu^{\prime}(x, t)$ and $\phi^{\prime}(x, t)$, which serve as inputs to the calculation of $e_{0}^{B F}(t)$ and $e_{0}^{\prime}(t)$; in both cases, a measure of age-specific quantum has been inflated (or deflated) by a factor of $\left(1-\bar{r}_{c}(t)\right)^{-1}$.

The close relationship between $e_{0}^{*}$ and $e_{0}^{B F}$, can be illustrated by re-writing equation 27 as follows:

$$
\begin{aligned}
e_{0}^{B F}(t) & =\int_{0}^{\infty} \exp \left\{-\int_{0}^{x} \frac{\mu(a, t)}{1-\bar{r}_{c}(t)} d a\right\} d x \\
& =\int_{0}^{\infty} \exp \left\{-\int_{0}^{x} \frac{1-r_{c}(x, t-x)}{1-\bar{r}_{c}(t)} \mu^{*}(a, t) d a\right\} d x .
\end{aligned}
$$

Similarly, we can see the resemblance between $e_{0}^{*}$ and $e_{0}^{\prime}$ by re-writing equation 24 as follows:

$$
e_{0}^{\prime}(t)=\int_{0}^{\infty} x \frac{\phi_{c}(x, t-x)}{1-\bar{r}_{c}(t)} d x=\int_{0}^{\infty} x \frac{1-r_{c}(x, t-x)}{1-\bar{r}_{c}(t)} \phi^{*}(x, t) d x
$$


In both of the above formulas, the ratio of $1-r_{c}(x, t-x)$ to $1-\bar{r}_{c}(t)$ will be close to one so long as $r_{c}(x, t-x)$ does not vary widely as a function of age at time $t$. Bongaarts and Feeney $(2002,2003)$ assume that $r_{c}(x, t-x)$ is indeed constant with age (their "proportionality" assumption). Since reality resembles this assumption in some cases, the three measures are sometimes approximately equal. However, Guillot (2003) notes that observed differences between $C A L$ and $e_{0}^{\prime}$ can be substantial: for French males the difference was 2.51 years in 2001 and was even larger in earlier decades (e.g., 9.24 years in 1954).

According to Bongaarts and Feeney $(2002,2003)$, these two quantities, $\mu^{\prime}(x, t)$ and $\phi^{\prime}(x, t)$, are "tempo-adjusted" mortality functions. However, unlike $\mu^{*}(x, t)$ and $\phi^{*}(x, t), \mu^{\prime}(x, t)$ and $\phi^{\prime}(x, t)$ are not in general associated with the same probability of survival to age $x$. That is,

$$
\begin{aligned}
\int_{x}^{\infty} \phi^{\prime}(a, t) d a & =\int_{x}^{\infty} \frac{\phi_{c}(a, t-a)}{1-\bar{r}_{c}(t)} d a=\frac{\int_{x}^{\infty}\left(1-r_{c}(a, t-a)\right) \phi^{*}(a, t) d a}{1-\bar{r}_{c}(t)} \\
& =\frac{\int_{x}^{\infty} \phi^{*}(a, t) d x-\int_{x}^{\infty} r_{c}(a, t-a) \phi^{*}(a, t) d a}{1-\bar{r}_{c}(t)}=\ell_{c}(x, t-x) \frac{1-\bar{r}_{c}\left(x^{+}, t\right)}{1-\bar{r}_{c}(t)},
\end{aligned}
$$

where $\bar{r}_{c}\left(x^{+}, t\right)=\int_{x}^{\infty} r_{c}(a, t-a) \frac{\phi^{*}(a, t)}{\ell_{c}(x, t-x)} d a$ by definition. In contrast,

$$
\exp \left\{-\int_{0}^{x} \mu^{\prime}(a, t) d a\right\}=\exp \left\{-\int_{0}^{x} \frac{\mu(a, t)}{1-\bar{r}_{c}(t)} d a\right\}=\ell_{p}(x, t)^{\left(1-\bar{r}_{c}(t)\right)^{-1}} .
$$

Thus, these two quantities are not the same in general, and for this reason $e_{0}^{B F}(t)$ and $e_{0}^{\prime}(t)$ are not equal except under special circumstances: when $s_{c}(x, t-x)$ and $r_{c}(x, t-x)$ are constant as a function of age $x$ (for a given time $t$ ).

Furthermore, as noted also by Feeney $(2004,2005)$, the general form of tempoadjusted mortality should involve an appropriate adjustment at each age, not an average correction applied uniformly across the age range. Although Feeney's notation was different, he also proposed an age-specific adjustment factor of $1+s_{c}(x, t-x)=\left(1-r_{c}(x, t-x)\right)^{-1}$. As noted earlier in the definitions of $\mu^{*}(x, t)$ and $\phi^{*}(x, t)$ used here (see equations 16 and 19), this adjustment to observed cohort 
mortality functions reflects the shift in the cohort distribution of age at death occurring at that exact age and time, rather than some average value across all ages at time $t$. For comparison, note the factor of $\left(1-\bar{r}_{c}(t)\right)^{-1}$ used in the definition of $\mu^{\prime}(x, t)$ and $\phi^{\prime}(x, t)$.

As shown earlier, $e_{0}^{*}$ (or $C A L$ ) can be computed from either $\mu^{*}(x, t)$ or $\phi^{*}(x, t)$. Thus, $C A L$ is exactly equal to the generalized form of tempo-adjusted life expectancy at birth proposed by Feeney $(2004,2005)$. In the equations for $e_{0}^{*}$, the factor of $1+s_{c}(x, t-x)$, or $\left(1-r_{c}(x, t-x)\right)^{-1}$, replaces the lost quantum at age $x$ that results from delay in the timing of mortality from cohort to cohort. The substantive value of this particular interpretation of $C A L$ is dubious (see later discussion). Nevertheless, if this concept is useful at all, then it is worth noting that the generalized form of tempoadjusted life expectancy at birth equals $e_{0}^{*}$, or $C A L$, which is different from the original measure proposed under the same label by Bongaarts and Feeney. In fact, $e_{0}^{B F}$ differs in general from both $e_{0}^{*}$ and $e_{0}^{\prime}$. As noted earlier, the latter two measures provide interesting descriptions of population dynamics (in a constant-birth population). Except in the special case when it equals these other two measures, $e_{0}^{B F}$ seems to have no substantively interesting interpretation on its own.

\subsection{Comparison to period life expectancy at birth, $e_{0}$}

Even in the special case where $e_{0}^{*}, e_{0}^{\prime}$, and $e_{0}^{B F}$ are equivalent, their value is typically different from the period life expectancy at birth, $e_{0}$. The latter measure would equal the other three only if the age pattern of mortality were constant over time. However, in the case of a sustained mortality decline, $e_{0}$ tends to be higher than the other measures. Let us consider why this difference occurs, focusing in particular on a comparison between $e_{0}$ and $e_{0}^{*}$.

Because $e_{0}(t)$ and $e_{0}^{*}(t)$ equal the sum of period and cohort survival probabilities across the age range at time $t$ (see equations 1 and 22), it is useful to understand the relationship between $\ell_{p}(x, t)$ and $\ell_{c}(x, t-x)$. As noted earlier, the period survival probability, $\ell_{p}(x, t)$, is a function of death rates observed at time $t$ alone. Likewise, the associated cohort survival probability, $\ell_{c}(x, t-x)$, depends only on death rates along a 
single diagonal lifeline of the Lexis diagram. However, such diagonals pertain to different time periods even for a single cohort, and to a wide interval of age and time when we consider the collection of cohorts alive at time $t$. Thus, an important difference between $e_{0}(t)$ and $e_{0}^{*}(t)$ is that the former is a function of death rates for time $t$ alone, whereas the latter depends on all death rates (past and present) experienced by cohorts alive at time $t$.

Furthermore, it is possible to show that

$$
\ell_{c}(x, t-x)=\ell_{p}(x, t) \exp \left\{\int_{0}^{x} \int_{0}^{a} \frac{d}{d t} \mu(y, t-a+y) d y d a\right\} .
$$

In this equation, the argument to the exponential function is merely the total change in death rates occurring within the triangle of the Lexis diagram that lies below the diagonal lifeline of the cohort born at time $t-x$, and to the left of a vertical line at time $t$. Thus, in a situation of sustained mortality decline, the survival probability for a cohort, $\ell_{c}(x, t-x)$, would be higher than its corresponding period value, $\ell_{p}(x, t)$, by a factor that depends on the total reduction in mortality risks over this triangular interval of age and time. This representation leads to a useful interpretation of observed differences between $e_{0}$ and $e_{0}^{*}$.

As noted already, in a situation of sustained mortality decline, $e_{0}^{*}(t)$ tends to be lower than $e_{0}(t)$, because $\ell_{c}(x, t-x)$ tends to be lower than $\ell_{p}(x, t)$ across age, $x$, for a fixed time, $t$. Using equation 32 , it is possible to convert $\ell_{p}(x, t)$ into $\ell_{c}(x, t-x)$ simply by factoring out the gains in period survival probabilities due to historical reductions in mortality rates. Likewise, $e_{0}^{*}(t)$ is lower than $e_{0}(t)$ in this situation because it also does not take into account these past improvements in mortality. This analysis illustrates why the concept of "tempo-adjusted" life expectancy has little value. For a measure of tempo such as $e_{0}$, an adjustment designed to remove the impact of "tempo change" also has the effect of erasing some of the gains in longevity implied by historical reductions in age-specific death rates. For this reason, both $e_{0}^{*}$ and $e_{0}^{\prime}$ are useful primarily as measures of population dynamics (see earlier discussion). 


\section{Trends in life expectancy at birth by period and cohort}

\subsection{Speed of change in historical trends}

Figures $3 \mathrm{~A}$ and $3 \mathrm{~B}$ show actual and smoothed trends in period and cohort life expectancy at birth, plotted in the usual way (by year of death for period $e_{0}$, and year of birth for cohort $e_{0}$ ). Then, for comparison with period $e_{0}$, Figure 3C shows the smoothed trend in Swedish cohort $e_{0}$ plotted in two ways: by year of birth, and in relation to the time when the cohort's mean age at death actually occurs. ${ }^{8}$ Note that the slope of the cohort trend tends to be greater than the slope of the period trend when cohort $e_{0}$ is plotted as a function of year of birth, but less when plotted according to the period in which the cohort mean age at death actually occurs.

\section{Figure 3: Life expectancy at birth in Sweden}

A) Periods, actual vs. smoothed trends, 1751-2002

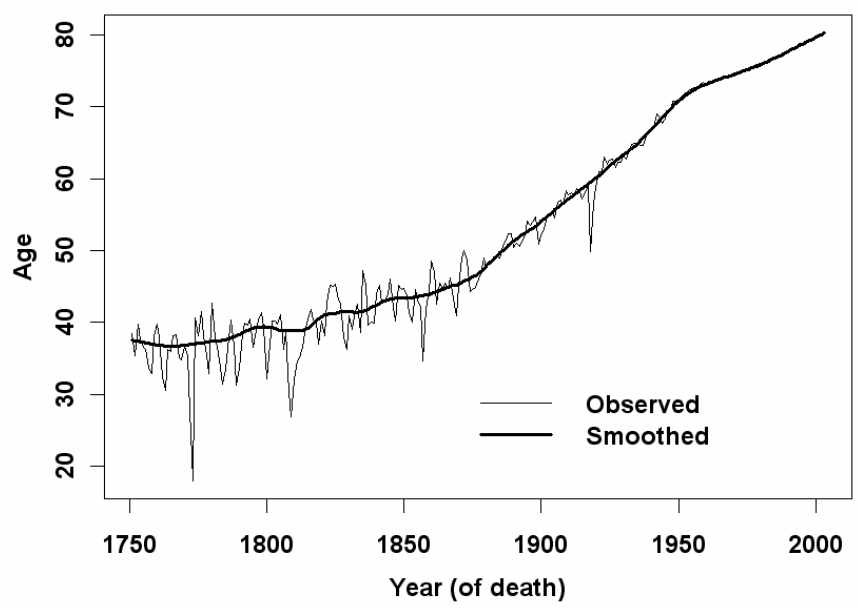

Note: The observed trend was smoothed using the LOWESS method (Chambers et al., 1983).

Source: Human Mortality Database (2004).

\footnotetext{
${ }^{8}$ Bongaarts (2005) refers to the latter measure as "lagged cohort life expectancy." The time lag in this case is the cohort life expectancy itself.
} 
Wilmoth: On the relationship between period and cohort mortality

\section{Figure 3: Continued}

B) Cohorts, actual vs. smoothed trends, 1751-1911

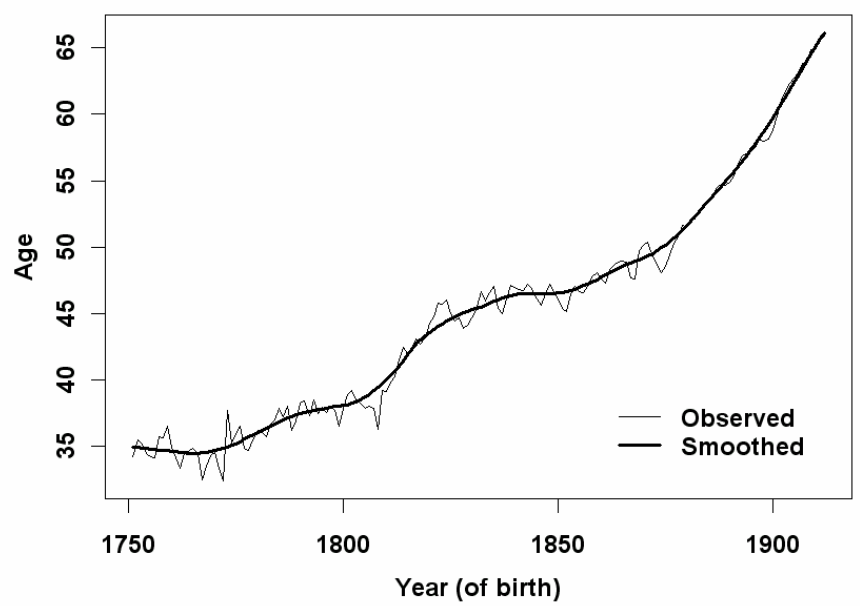

Notes: (1) See note for Figure 3A. (2) Data employed here for cohorts born after approx. 1890 are incomplete. Therefore, estimates of life expectancy at birth for these cohorts rely on recent period data at very high ages (i.e., above age 90 ). Source: Human Mortality Database (2004).

C) Periods vs. cohorts, smoothed trends only

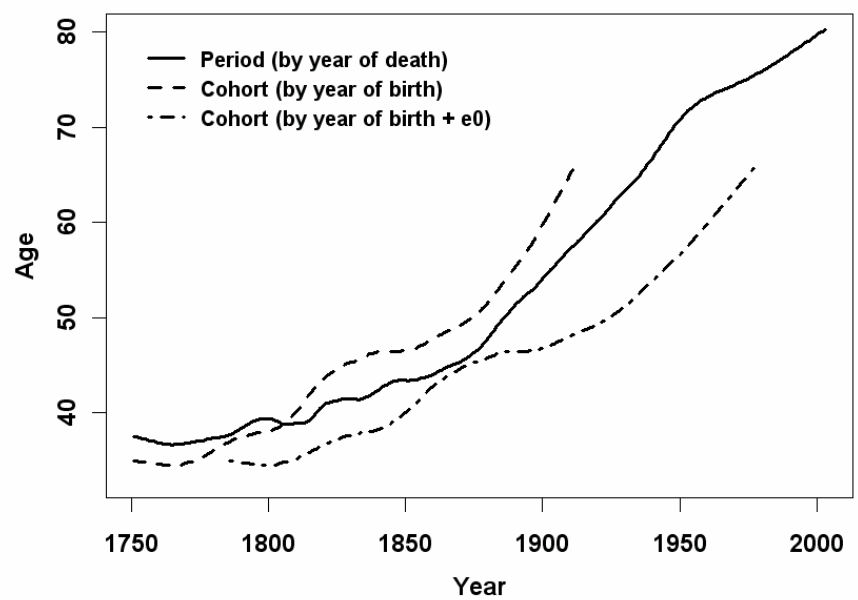

Notes: See notes for Figure 3B.

Source: Human Mortality Database (2004). 
In part, such differences are due to fluctuations over time in historical mortality trends, which affect the mean lifespan of periods and cohorts in complicated ways. Such factors are beyond the scope of the present work. However, in addition to the arbitrary influences of history, there exists an intrinsic difference between period and cohort trends in $e_{0}$ due to the fundamental mathematical relationship linking the age and time of death to a decedent's time of birth.

\subsection{Intrinsic difference in period-cohort trends}

As before, let $\tau=t-x$. In words, cohort $=$ period - age . Clearly, when $x$ is fixed, $d \tau=d t$. However, when $x$ is changing, $d \tau=d t-d x$. In that case,

$$
\frac{d \tau}{d t}=1-\frac{d x}{d t}=1-r \quad \text { and } \quad \frac{d t}{d \tau}=1+\frac{d x}{d \tau}=1+s .
$$

where $r=\frac{d x}{d t}$ and $s=\frac{d x}{d \tau}$. Therefore,

$$
1-\frac{d x}{d t}=\left(1+\frac{d x}{d \tau}\right)^{-1} \quad \text { or } \quad 1-r=(1+s)^{-1} .
$$

It also follows that

$$
r=\frac{s}{1+s} \quad \text { and } \quad s=\frac{r}{1-r} .
$$

Thus, $r$ and $s$ represent two different measures of the speed of change over time in some function of age. The former is a slope with respect to the timing of the event itself, whereas the latter is with respect to the timing of birth for the cohort that experiences the event. This relationship is valid for any life course event (not only death) and was noted previously by Zeng and Land (2002) in the case of fertility.

Figure 4 offers a simple example: a trend in which some measure of tempo increases by 1 year of age over 5 years of time (thus, $r=0.2$ ). However, the same increase involves only 4 cohorts (thus, $s=0.25$ ). 
Figure 4: Simple example illustrating intrinsic difference in slope of age trend from perspective of periods $(r=0.2)$ and cohorts $(s=0.25)$

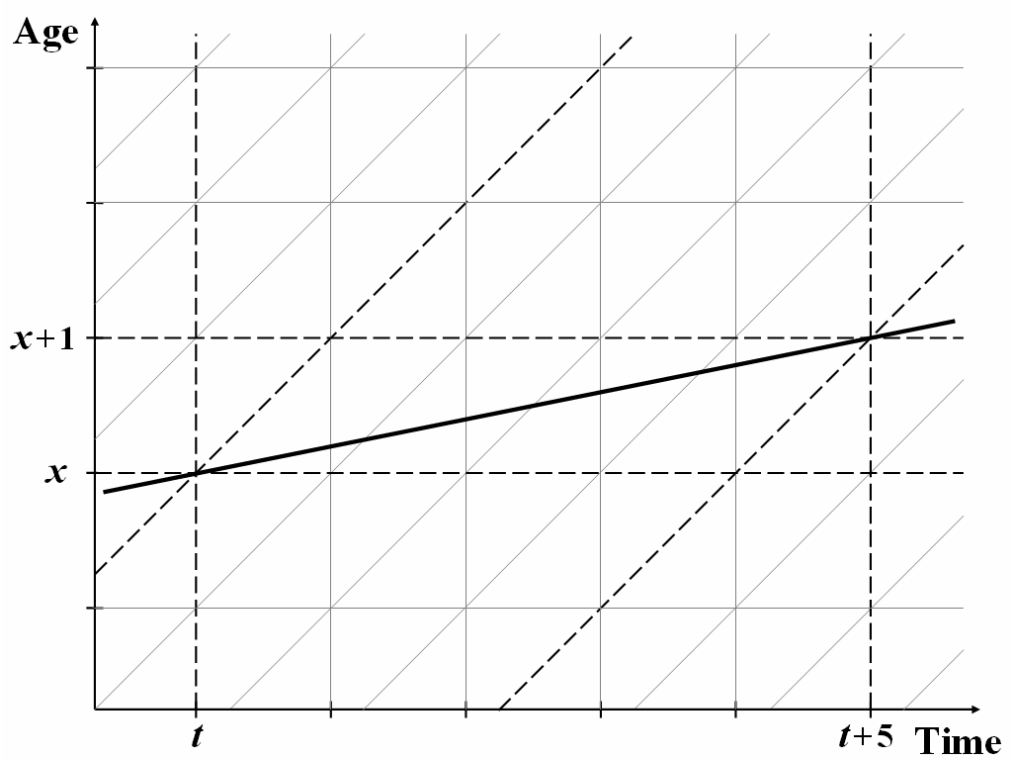

\subsection{Period-cohort trends in the linear shift model}

In order to elucidate the relationship between period and cohort mortality, it is useful to simulate historical trends using a model of a shifting distribution of age at death. The shift model explored here has three important characteristics:

a) It is linear (i.e., the trend in each percentile of the distribution is linear over time);

b) It is sustained over a long duration (i.e., the shift extends relatively far into both the past and the future); and

c) It is defined in relation to a baseline mortality distribution associated with time $t=0 .{ }^{9}$

\footnotetext{
${ }^{9}$ Time $t=0$ is chosen as the baseline for the model in order to keep the formulas as simple as possible. If one wishes to use some other year, say $t_{0}$, as the reference point for the shift, then all formulas shown here could be modified by substituting $t^{\prime}=t-t_{0}$ in place of $t$.
} 
To simplify the exposition, the linear shift model described here is specified in terms of period mortality at time $t=0$. It is also possible to define such a model as a function of cohort mortality at time $t=0$ (i.e., based on a cross-section of cohort mortality distributions at this moment). However, as shown in the Appendix (section A-2.2), a sustained linear shift model yields identical results for those periods and cohorts whose lifespans lie fully within the shift whether the model is defined in terms of period or cohort mortality. ${ }^{10}$ Therefore, I assume here that the time scale of the shift is relatively long (say, 150 years both forward and backward from time $t=0$ ).

As was done earlier for cohorts, let us define percentiles of the period distribution of age at death (i.e., for the synthetic cohort associated with period $t$ ) as follows:

$$
\tilde{a}_{p}(\pi, t)=x \quad \text { such that } \quad \pi=\Phi_{p}(x, t)=1-\ell_{p}(x, t),
$$

where $\Phi_{p}(x, t)=\int_{0}^{x} \phi_{p}(a, t) d a$ is the distribution (or cumulative probability) function for age at death in period $t$. Furthermore, assume that the percentile associated with the same value of $\pi$ equals $y$ at time $t=0$ :

$$
\tilde{a}_{p}(\pi, 0)=y \quad \text { such that } \quad \pi=\Phi_{p}(y, 0)=1-\ell_{p}(y, 0) .
$$

The relationship between these two ages, $x$ and $y$, can be used to specify the form of historical changes in the age distribution of deaths.

For example, the core assumption of the linear shift model is that the values of $x$ associated with a given $y$ form a straight line, whose slope may vary as a function of age:

$$
x=y+r(y) t \quad \text { for }-T<t<T,
$$

where $r(y)$ can take on different values as a function of age, $y$, subject to certain restrictions (see Appendix, section A-2.3); and $T$ is the duration of the shift both forward and backward from $t=0$. In general, let us assume that $T$ is sufficiently large to ensure that all cohorts alive at $t=0$ experience the shift for their entire lives. ${ }^{11}$

\footnotetext{
${ }^{10}$ Note that if the model involves an abrupt change of slope in the percentiles of a mortality distribution at some moment close to the present, say $t=0$, then there are important differences between these two approaches.

${ }^{11}$ As a practical matter, we can assume that all cohort lifespans are finite, and thus that some finite interval, from $-T$ to $T$, can contain the lifespans of all currently living cohorts. If we allow for theoretically infinite lifespans, $T$ needs to be large enough to assure that a very high proportion of deaths (say, $1-\varepsilon$, where $\varepsilon>0$ is very small) for cohorts alive at time $t=0$ occur during the period of the shift.
} 
Note that $\Phi_{p}(x, t)=1-\ell_{p}(x, t)=\pi$ is constant for all combinations of $x$ and $t$ along this percentile contour line. Therefore, another way of describing the core assumption of a linear shift model is that

$$
\Phi_{p}(x, t)=\Phi(y) \quad \text { or } \quad \ell_{p}(x, t)=\ell(y),
$$

where $y=x-r(y) t$. Thus, $\Phi(y)$ and $\ell(y)$ depict the baseline mortality distribution and survival probabilities for the linear shift model. They are identical to the corresponding period mortality functions associated with time $t=0$ in the model (i.e., $\Phi_{p}(y, 0)=\Phi(y)$ and $\left.\ell_{p}(y, 0)=\ell(y)\right)$.

It is shown in the Appendix (section A-2.1) that in a sustained linear shift model, period life expectancy at birth during the shift interval (i.e., for $-T<t<T$ ) has the following form:

$$
e_{0}^{p}(t)=e_{0}+\bar{r} t
$$

where $e_{0}=\int_{0}^{\infty} x \phi(x) d x ; \bar{r}=\int_{0}^{\infty} r(x) \phi(x) d x$; and $\phi(x)=\frac{d}{d x} \Phi(x)=-\frac{d}{d x} \ell(x)$. In the same model, life expectancy at birth for the cohort born in year $\tau$ is as follows:

$$
e_{0}^{c}(\tau)=e_{0}^{*}+\int_{0}^{\infty} x s(x) \phi^{*}(x) d x+\bar{s} \tau=\int_{0}^{\infty} x(1+s(x)) \phi^{*}(x) d x+\bar{s} \tau,
$$

where $e_{0}^{*}=\int_{0}^{\infty} x \phi^{*}(x) d x ; \quad \bar{s}=\int_{0}^{\infty} s(x) \phi^{*}(x) d x ; s(x)=\frac{r(x)}{1-r(x)} ; \quad \mu^{*}(x)=\frac{\mu(x)}{1-r(x)} ;$ $\ell^{*}(x)=e^{-\int_{0}^{x} \mu^{*}(a) d a}$; and $\phi^{*}(x)=\ell^{*}(x) \mu^{*}(x)$. However, note that equation 41 applies only to those cohorts whose observed (finite) lifespan lies fully within the interval of the period-based shift (i.e., within $-T<t<T$ ).

Thus, period life expectancy at time $t=0$ serves as the baseline value for the linear shift model, i.e., $e_{0}^{p}(0)=e_{0}$. Let us consider the relationship between this quantity and cohort life expectancy for two particular cohorts:

a) The cohort born at that moment, i.e., $\tau=0$; and

b) The cohort whose average age at death occurs at time $t=0$. 
As indicated by equation 41 above, cohort life expectancy is a function of $e_{0}^{*}$, or $C A L$, at time $t=0$. For the cohort born at time $\tau=0$, this equation simplifies to the following:

$$
e_{0}^{c}(0)=\int_{0}^{\infty} x(1+s(x)) \phi^{*}(x) d x=e_{0}^{*}+\int_{0}^{\infty} x s(x) \phi^{*}(x) d x
$$

However, case b) is more complicated.

Obviously the cohort whose average age at death occurs at time $t=0$ must have been born at some earlier date, say $\tau=-\lambda$, where $\lambda>0$. Setting $e_{0}^{c}(-\lambda)=\lambda$ in equation 41 and then solving for $\lambda$ yields the following formula:

$$
e_{0}^{c}(-\lambda)=\lambda=\int_{0}^{\infty} x \frac{1+s(x)}{1+\bar{s}} \phi^{*}(x) d x=\frac{e_{0}^{c}(0)}{1+\bar{s}}
$$

Therefore, the mean ages at death for these two cohorts differ by a factor of $1+\bar{s}$. Also, note that if $s(x)$ is close to constant over the age range, then $(1+s(x)) /(1+\bar{s})$ will be close to one. In that case, $e_{0}^{c}(-\lambda)=\lambda$ would have a similar value to $e_{0}^{*}$. Thus, as a rough approximation, $C A L(0)$ equals the cohort mean age at death that is attained at time $t=0$ by a cohort born $C A L(0)$ years earlier, assuming linear trends over time in cohort percentiles of age at death.

\subsection{Empirical application of the linear shift model}

For empirical applications of the linear shift model, we redefine the origin of the time axis in each case so that the current year $t$ is treated as time 0 in the above formulas. The formulas given above for $e_{0}^{c}(0)$ and $e_{0}^{c}(-\lambda)=\lambda$ in this model provide motivation for two additional measures of mean lifespan based on cross-sectional cohort mortality patterns at time $t$. In the first case, define

$$
e_{0}^{* *}(t)=\int_{0}^{\infty} x\left(1+s_{c}(x, t-x)\right) \phi^{*}(x, t) d x=e_{0}^{*}(t)+\int_{0}^{\infty} x s_{c}(x, t-x) \phi^{*}(x, t) d x
$$


Comparing this formula to equation 42 above, it follows that $e_{0}^{* *}(t)$ equals the average age at death (or life expectancy) for the cohort born at time $t$, assuming linear trends in cross-sectional cohort percentiles of age at death. In other words, if we create a linear shift model using cohort (not period) mortality patterns observed at time $t$, the resulting estimate of $e_{0}^{c}(t)$ equals $e_{0}^{* *}(t)$.

In the second case, let

$$
e_{0}^{\prime \prime}(t)=\int_{0}^{\infty} x \frac{1+s_{c}(x, t-x)}{1+\bar{s}_{c}(t)} \phi^{*}(x, t) d x=\frac{e_{0}^{* *}(t)}{1+\bar{s}_{c}(t)}
$$

Like $e_{0}^{* *}(t)$, this quantity is a linear projection of cross-sectional cohort mortality at time $t$. If historical changes mimic the linear shift model exactly, then $e_{0}^{\prime \prime}(t)=e_{0}^{c}(t-\lambda)=\lambda$ (compare equation 43 above). Furthermore, even when actual conditions differ from assumptions of the this model, $e_{0}^{\prime \prime}(t)$ may serve as a useful approximation of cohort life expectancy for the cohort whose average age at death occurs at time $t$.

In Figure 5, smoothed trends in Swedish period and cohort life expectancy at birth are compared to these two sets of predictions, $e_{0}^{* *}(t)$ and $e_{0}^{\prime \prime}(t)$. These results demonstrate that the predictions of the linear shift model match reality reasonably well. Therefore, it seems to be possible to use this model to form plausible statements about mortality for individual cohorts based on mortality patterns for the collection of cohorts observed in a cross-section at a moment of time. However, the current purpose of these calculations is not to obtain estimates or forecasts of cohort life expectancy, but rather to provide insights into the relationship between period and cohort mortality. Note that each value of $e_{0}^{* *}(t)$ and $e_{0}^{\prime \prime}(t)$ in Figure 5 is based on cohort mortality patterns for a single year. For this reason, the period of unusually rapid mortality change around the middle of the $20^{\text {th }}$ century produces exaggerated trends in both cases (over-estimating the mean lifespan for cohorts born in those years, and under-estimating it for the cohorts whose mean age at death occurs in those years).

Furthermore, note that

$$
C A L(t)=e_{0}^{*}(t) \approx \frac{1}{2}\left(e_{0}^{\prime}(t)+e_{0}^{\prime \prime}(t)\right)
$$


since

$$
\frac{1}{2}\left(\frac{1-r_{c}(x, t-x)}{1-\bar{r}_{c}(t)}+\frac{1+s_{c}(x, t-x)}{1+\bar{s}_{c}(t)}\right) \approx 1,
$$

at least in the case of a smooth mortality surface (see next paragraph). In the special case where $r_{c}(x, t-x)=r$ and $s_{c}(x, t-x)=s$ for all $x$, these relationships are exact, as the three measure are identical in this situation. The approximate symmetry of these three quantities over time (from 1861 until 2003, for the national population of Sweden) can be seen in Figure 6, which also shows empirical trends in $e_{0}$ and $e_{0}^{B F}$ (for 6A), or $e_{30}$ and $e_{30}^{B F}$ (for $6 \mathrm{~B}$ ).

Figure 5: Life expectancy at birth by period and cohort, plus estimates of cohort $e_{0}$ assuming linear trends in cross-sectional cohort percentiles of age at death, Sweden, 1751-2003

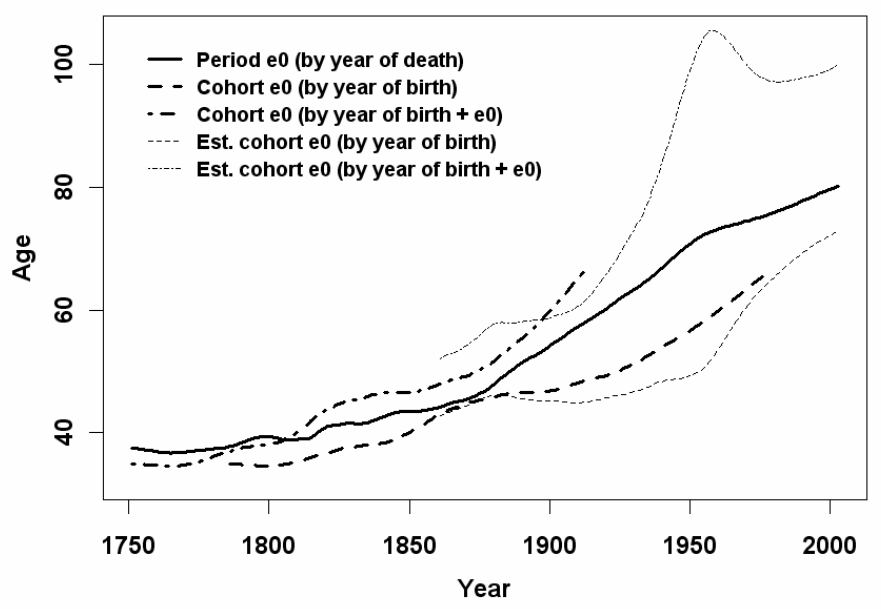


Figure 6: Period life expectancy plus four measures of mean age at death based on cross-sectional cohort mortality, Sweden, 1861-2003

A) At birth

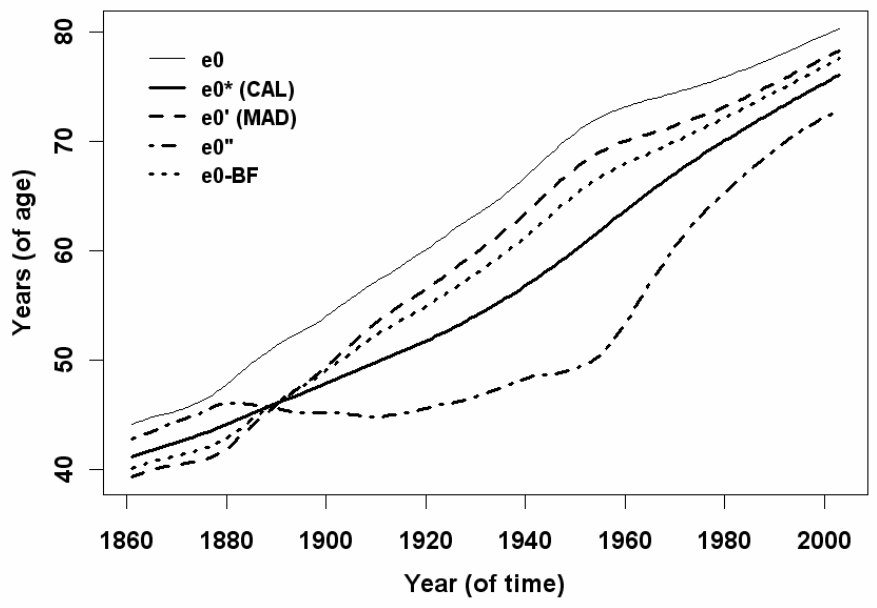

B) At age 30

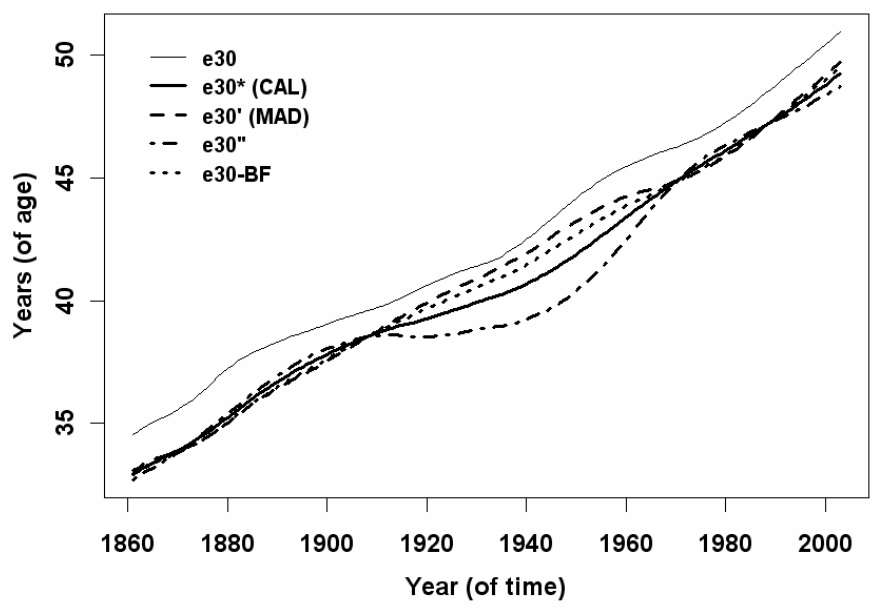

Note: The $y$-axis here represents the number of years beyond age 30 . In the case of $e_{30}$, this is known as remaining life expectancy. 
It is important to note that all estimates for the measures shown in Figures 5 and 6 were derived from smoothed surfaces of period and/or cohort survival probabilities (for single years of age and time). Although other smoothing techniques could have been used, the strategy employed here was to smooth the two empirical surfaces, $\ell_{p}(x, t)$ and $\ell_{c}(x, t-x)$, over time for each age group separately using the LOWESS procedure (Chambers et al., 1983), and then to compute all other quantities from the pair of smoothed survival surfaces. Although other techniques could be explored as well, a full treatment of this topic is beyond the scope of the present work.

For most of the measures used in this paper, including $e_{0}, e_{0}^{\prime} e_{0}^{*}$, and $e_{0}^{B F}$, the smoothing procedure merely removes short-term fluctuations in trends. However, for

$e_{0}^{\prime \prime}$ and $e_{0}^{* *}$, the results shown here would be fundamentally different (and clearly less plausible) if calculations were based on unsmoothed data. As noted before, these two measures give projected values of cohort life expectancy based on a linear shift model. Such calculations appear to be adversely affected by observed values of $s_{c}(x, t-x)$ less than -1 , which seem to arise only when using unsmoothed data. In such cases, the $\phi^{*}(x, t)$ and $\mu^{*}(x, t)$ functions take on negative values, and at the same time the approximations of equations 46 and 47 become unreliable. A comparison of estimates to empirical patterns shows clearly that the results based on smoothed data are superior. (Results shown here are based on smoothed data only; results based on unsmoothed data are available from the author on request.) Furthermore, there are clear theoretical motivations for using only smooth age patterns of mortality change (as depicted in agespecific percentile slopes) in empirical applications of the linear shift model (see Appendix, section A-2.3).

\section{Comparison of six measures of mean lifespan}

Table 1 compares the six measures of mean lifespan explored in this paper. Among these six measures, only life expectancy at birth, $e_{0}$, can be computed solely as a function of death rates at time $t$. All of the other measures depend on cohort survival probabilities at time $t, \ell_{c}(x, t-x)$, which reflect mortality conditions from the past as well as the present.

In addition to life expectancy at birth, the table also contains two measures, $e_{0}^{*}$ and $e_{0}^{\prime}$, that are based on a model of population dynamics in which the inflow of births is 
Wilmoth: On the relationship between period and cohort mortality

\section{Table 1: Summary of Six Measures of Mean Lifespan for Period $t$}

\begin{tabular}{|c|c|c|c|}
\hline Name & Notation & Equivalent forms & Short Description \\
\hline $\begin{array}{l}\text { Life expectancy } \\
\text { at birth }\end{array}$ & $e_{0}(t)$ & $\begin{aligned} \int_{0}^{\infty} & \ell_{p}(x, t) d x \\
& =\int_{0}^{\infty} x \phi_{p}(x, t) d x \\
& =\int_{0}^{\infty} \exp \left\{-\int_{0}^{x} \mu(a, t) d a\right\} d x\end{aligned}$ & $\begin{array}{l}\text { Mean age at death in a synthetic } \\
\text { cohort exposed throughout life to } \\
\text { the age-specific death rates of } \\
\text { time } t\end{array}$ \\
\hline $\begin{array}{l}\text { Cross-sectional } \\
\text { average length } \\
\text { of life }(C A L)\end{array}$ & $e_{0}^{*}(t)$ & $\begin{array}{l}\int_{0}^{\infty} \ell_{c}(x, t-x) d x \\
\quad=\int_{0}^{\infty} x \phi^{*}(x, t) d x \\
\quad=\int_{0}^{\infty} \exp \left\{-\int_{0}^{x} \mu^{*}(a, t) d a\right\} d x\end{array}$ & $\begin{array}{l}\text { Relative size of a population at } \\
\text { time } t \text { assuming a constant } \\
\text { inflow of births; also, the } \\
\text { generalized form of tempo- } \\
\text { adjusted } e_{0}\end{array}$ \\
\hline $\begin{array}{l}\text { Mean age at } \\
\text { death }(M A D)\end{array}$ & $e_{0}^{\prime}(t)$ & $\begin{array}{l}\frac{\int_{0}^{\infty} x \phi_{c}(x, t-x) d x}{\int_{0}^{\infty} \phi_{c}(x, t-x) d x} \\
\quad=\int_{0}^{\infty} x \phi^{\prime}(x, t) d x \\
\quad=\int_{0}^{\infty} x \frac{1-r_{c}(x, t-x)}{1-\bar{r}_{c}(t)} \phi^{*}(x, t) d x\end{array}$ & $\begin{array}{l}\text { Mean age at death in a } \\
\text { population at time } t \text { assuming a } \\
\text { constant inflow of births }\end{array}$ \\
\hline $\begin{array}{l}\text { "Tempo-adjusted" } \\
\text { life expectancy } \\
\text { at birth }\end{array}$ & $e_{0}^{B F}(t)$ & $\begin{array}{l}\int_{0}^{\infty} \exp \left\{-\int_{0}^{x} \mu^{\prime}(a, t) d a\right\} d x \\
\quad=\int_{0}^{\infty} \exp \left\{-\int_{0}^{x} \frac{\mu(a, t)}{1-\bar{r}_{c}} d a\right\} d x\end{array}$ & $\begin{array}{l}\text { Tempo-adjusted } e_{0} \text { assuming a } \\
\text { parallel shift at time } t \text { in cohort } \\
\text { distributions of age at death }\end{array}$ \\
\hline $\begin{array}{l}\text { Expected cohort } \\
\text { life expectancy } \\
\text { at birth }\end{array}$ & $e_{0}^{* k}(t)$ & $\begin{aligned} \int_{0}^{\infty} & x\left(1+s_{c}(x, t-x)\right) \phi^{*}(x, t) d x \\
& =\left(1+\bar{s}_{c}(t)\right) e_{0}^{\prime \prime}(t) \\
& =e_{0}^{*}(t)+\int_{0}^{\infty} x s_{c}(x, t-x) \phi^{*}(x, t) d x\end{aligned}$ & $\begin{array}{l}\text { Mean age at death for cohort } \\
\text { born at time } t \text { assuming a linear } \\
\text { shift over time in cohort } \\
\text { distributions of deaths by age } \\
\text { (see note 2) }\end{array}$ \\
\hline $\begin{array}{l}\text { Achieved cohort } \\
\text { life expectancy } \\
\text { at birth }\end{array}$ & $e_{0}^{\prime \prime}(t)$ & $\begin{aligned} \int_{0}^{\infty} x \frac{1+s_{c}(x, t-x)}{1+\bar{s}_{c}(t)} \phi^{*}(x, t) d x \\
=\frac{e_{0}^{* *}(t)}{1+\bar{s}_{c}(t)}\end{aligned}$ & $\begin{array}{l}\text { Mean age at death, } \lambda, \text { for } \\
\text { cohort born at time } t-\lambda \\
\text { assuming a linear shift in cohort } \\
\text { distributions of deaths by age } \\
\text { (see note } 2 \text { ) }\end{array}$ \\
\hline
\end{tabular}

Notes: (1) By definition: $\phi^{*}(x, t)=\frac{\phi_{c}(x, t-x)}{1-r_{c}(x, t-x)} ; \mu^{*}(x, t)=\frac{\mu(x, t)}{1-r_{c}(x, t-x)} ; \phi^{\prime}(x, t)=\frac{\phi_{c}(x, t-x)}{1-\bar{r}_{c}(t)} ; \mu^{\prime}(x, t)=\frac{\mu(x, t)}{1-\bar{r}_{c}(t)}$

$$
\bar{s}_{c}(t)=\int_{0}^{\infty} s_{c}(x, t-x) \phi^{*}(x, t) d x ; \text { and } \bar{r}_{c}(t)=\int_{0}^{\infty} r_{c}(x, t-x) \phi^{*}(x, t) d x .
$$

(2) For $e_{0}^{* *}$ and $e_{0}^{\prime \prime}$, the speed of the assumed linear shift is derived from observed age-specific changes in cohort distributions of deaths by age at time $t$, after smoothing the cohort survival surface to remove short-term fluctuations in trends (see end of Section 5.4). 
assumed to be constant over time. These two quantities, as well as $e_{0}^{B F}$, are functions of shifts at time $t$ in cohort distributions of age at death. Both $e_{0}^{*}$ and $e_{0}^{\prime}$ can be used to describe the characteristics at time $t$ of a (hypothetical) constant-birth population. Unlike the other two measures, $e_{0}^{B F}$, appears to have no clear and useful interpretation of its own, except in a special case where all three measures would be equal. Empirically, $e_{0}^{B F}$ tends to lie somewhere between $e_{0}^{*}$ and $e_{0}^{\prime}$.

Table 1 also includes two measures, $e_{0}^{* *}$ and $e_{0}^{\prime \prime}$, that are derived from a linear shift model of mortality change. Both $e_{0}^{* *}$ and $e_{0}^{\prime \prime}$ are projected values of the cohort mean age at death based on trends in cohort mortality functions that are observed at time $t$. The projections shown here are based on smoothed estimates of the shift in cohort mortality at time $t$. Thus, although they are insensitive to rapid, year-to-year fluctuations in mortality, they seem to follow temporal variations in mortality over longer durations (decades, etc.). These measures might be more useful as actual projections of cohort mortality if they were based on observed changes averaged over relatively longer time periods.

\section{Conclusion}

In this study, we have compared life expectancy at birth to five other measures of mean lifespan for a given time period, $t$. Life expectancy, $e_{0}$, is unique among these measures for the fact that it is solely a function of death rates observed at time $t$ among surviving cohorts. For this reason, it is a pure period measure of mean lifespan. The other five measures do not share this characteristic, as they belong to the class of crosssectional cohort measures, which depend not only on cohort death rates at time $t$, but also on cohort survival probabilities observed at that moment. Since cohort survival probabilities are a function of death rates from periods before time $t$ as well, these measures form a fundamentally different class compared to pure period measures such as life expectancy at birth.

The concept of "tempo-adjusted" life expectancy forms a special case of the crosssectional cohort measures. Despite recent interest in this topic, the interpretive value of such a measure is not at all clear. In its generalized form, tempo-adjusted life expectancy at birth is equivalent to a measure known as CAL, or $e_{0}^{*}$ (Brouard, 1986; Guillot, 2003). In a special case, it also equals $e_{0}^{B F}$ (Bongaarts and Feeney, 2002, 
2003), as well as two other measures studied here, $e_{0}^{\prime}$ and $e_{0}^{\prime \prime}$. However, "tempo adjustment" in the case of $e_{0}$ appears to be synonymous with "removal of past changes in mortality rates." Survival probabilities computed from tempo-adjusted death rates (applying the general form of the concept) are equivalent to cross-sectional cohort survival probabilities. By substituting cohort for period survival probabilities in the calculation of life expectancy at birth, tempo-adjusted $e_{0}$ does not take into account all of the changes in mortality risks that have taken place during the lifetimes of living cohorts.

Because it lacks a useful general interpretation, the Bongaarts-Feeney measure $\left(e_{0}^{B F}\right)$ is the least interesting of the cross-sectional cohort measures considered here. Although it may sometimes be approximately equal to three of the other measures $\left(e_{0}^{*}\right.$, $e_{0}^{\prime}$, and $e_{0}^{\prime \prime}$ ), the latter quantities are no more difficult to calculate and correspond directly to the underlying theoretical constructs. Of the other four measures from the same class, two describe population dynamics at time $t$ based on a model of constant births $\left(e_{0}^{*}\right.$ and $\left.e_{0}^{\prime}\right)$. The other two are projected estimates of the mean age at death (or life expectancy at birth) for two particular cohorts $\left(e_{0}^{* *}\right.$ and $\left.e_{0}^{\prime \prime}\right)$; they can be derived using a linear shift model of mortality change (in which the percentiles of cohort distributions of age at death follow linear trends over time).

Analysis of empirical trends as well as the linear shift model has led to a pair of key insights concerning the relationship between period and cohort mortality. The first is that trends in period life expectancy at birth misrepresent the lived experience of cohorts in terms of the speed of change in the average length of life. In a situation of sustained mortality decline, for example, the rise in life expectancy over time tends to be slightly faster from cohort to cohort than from period to period. The second is that period life expectancy at birth for time $t$ tends to lie between the mean ages at death for two special cohorts: the cohort born at time $t$, and the cohort whose mean age at death is attained at time $t$ (two of the measures investigated here, $e_{0}^{* *}$ and $e_{0}^{\prime \prime}$, are projected estimates of these two quantities).

\section{Acknowledgements}

The author wishes to thank Michel Guillot, Alberto Palloni, and three anonymous reviewers for their comments on an earlier version. This research was supported by a grant from the National Institute on Aging, R01 AG11552. 


\section{References}

Bongaarts, John, and Griffith Feeney (1998). "On the quantum and tempo of fertility." Population and Development Review 24(2): 271-291.

Bongaarts, John, and Griffith Feeney (2002). "How long do we live?" Population and Development Review 28(1): 13-29.

Bongaarts, John, and Griffith Feeney (2003). "Estimating mean lifetime." Proceedings of the National Academy of Sciences 100(23): 13127-13133.

Bongaarts, John (2005). "Five period measures of longevity." Demographic Research, forthcoming.

Brouard, Nicolas (1986). "Structure et dynamique des populations. La pyramide des années à vivre, aspects nationaux et exemples régionaux." Espace, Populations, Sociétés (2): 157-168.

Calot, Gérard (2001). 'Mais qu'est-ce donc qu'un indicateur conjoncturel de fécondité?" Population 56(3): 325-327.

Chambers, John M., William S. Cleveland, and Paul A. Tukey (1983). Graphical Methods for Data Analysis. Boston: Duxbury.

Feeney, Griffith (2004). "A generalized mortality tempo adjustment formula." Workshop on Tempo Effects on Mortality, New York, November 18-19.

Feeney, Griffith (2005). "Increments to life and mortality tempo." Demographic Research, forthcoming.

Guillot, Michel (2003). "The cross-sectional average length of life (CAL): A crosssectional mortality measure that reflects the experience of cohorts." Population Studies 57(1): 41-54.

Guillot, Michel (2005). "Tempo effects in mortality: An appraisal." Demographic Research, forthcoming.

Hajnal, John (1947). "The analysis of birth statistics in the light of the recent international recovery of the birth-rate.” Population Studies 1(2): 137-164.

Human Mortality Database (2004). University of California, Berkeley (USA), and Max Planck Institute for Demographic Research (Germany), available at www.mortality.org or www.humanmortality.de (data downloaded July 27, 2004). 
Ryder, Norman B. (1964). "The process of demographic translation." Demography 1(1): 74-82.

Ryder, Norman B. (1978). "A model of fertility by planning status." Demography 15(4): 433-458.

Schoen, Robert (2004). "Timing effects and the interpretation of period fertility." Demography 41(4): 801-819.

Zeng, Yi, and Kenneth C. Land (2002). "Adjusting period tempo changes with an extension of Ryder's basic translation equation.” Demography 39(2): 269-285. 


\section{Appendix}

\section{A-1. Percentile slopes of cohort distributions of age at death}

Recall from the main text that percentiles of the distribution of age at death for the cohort born at time $\tau$ are defined as follows:

$$
\tilde{a}_{c}(\pi, \tau)=x \quad \text { such that } \quad \pi=\Phi_{c}(x, \tau)=1-\ell_{c}(x, \tau),
$$

where $\Phi_{c}(x, \tau)=\int_{0}^{x} \phi_{c}(a, \tau) d a$ is the distribution (or cumulative probability) function for age at death in the given cohort. Also recall the following definition for the pace of change (from cohort to cohort) in the percentile of this distribution that occurs at age $x$ :

$$
s_{c}(x, \tau)=\frac{d}{d \tau} \tilde{a}_{c}(\pi, \tau),
$$

where $\pi=\Phi_{c}(x, \tau)$ is fixed. I show here that this cohort percentile slope has the following equivalent forms:

$$
s_{c}(x, \tau)=\frac{-\frac{d}{d \tau} \Phi_{c}(x, \tau)}{\phi_{c}(x, \tau)}=\frac{\frac{d}{d \tau} \ell_{c}(x, \tau)}{\phi_{c}(x, \tau)}=\frac{\frac{d}{d \tau} \ln \ell_{c}(x, \tau)}{\mu_{c}(x, \tau)}=\frac{-\frac{d}{d \tau} H_{c}(x, \tau)}{\mu_{c}(x, \tau)} .
$$

For simplicity, let us consider a single age $x$ for the cohort born at time $\tau$, and let $s=s_{c}(x, \tau)$. As noted by Bongaarts and Feeney (2002), in order for $s$ to equal the slope of the percentile associated with age $x$ for the given cohort, it must satisfy the following equation:

$$
\left.\frac{d}{d a} \Phi_{c}(x+s a, \tau+a)\right|_{a=0}=0 .
$$

That is, a change of $s$ units in $x$ accompanied by a unit change in $\tau$ is associated with no change whatsoever in the cumulative probability of death, $\pi$, in the immediate vicinity of $x$ and $\tau$. Let $y=x+s a$ and $u=\tau+a$. It follows that:

$$
\frac{d}{d a} \Phi_{c}(x+s a, \tau+a)=\frac{d}{d y} \Phi_{c}(y, u) \frac{d y}{d a}+\frac{d}{d u} \Phi_{c}(y, u) \frac{d u}{d a}=\phi_{c}(y, u) s+\frac{d}{d u} \Phi_{c}(y, u) .
$$


Setting $a=0$ and equating the result to zero gives us the following expression:

$$
\phi_{c}(x, \tau) s+\frac{d}{d \tau} \Phi_{c}(x, \tau)=0
$$

Solving for $s$ yields the first relationship in equation 11. The other three forms of $s$ follow directly from the following elementary relationships: (a) $\ell_{c}(x, \tau)=-\Phi_{c}(x, \tau)$; (b) $\ln \ell_{c}(x, \tau)=-H_{c}(x, \tau)$; and (c) $\phi_{c}(x, \tau)=\ell_{c}(x, \tau) \mu_{c}(x, \tau)$.

\section{A-2. Fundamental properties of the linear shift model}

Equations 40 and 41 describe the trend in period and cohort life expectancy at birth in the case of a linear shift model (subject to the conditions stated at the beginning of section 5.3). In this section of the appendix, I derive these two equations and demonstrate that equivalent results are obtained whether a linear shift model is specified in terms of period or cohort mortality. I also discuss some necessary restriction on the possible values for percentile slopes within a linear shift model.

\section{A-2.1 Period and cohort life expectancy at birth}

As stated in the main text, a linear shift model can be specified by assuming that each percentile of the period distribution of age at death (i.e., within successive period life tables for the model population) is constant along a line defined by the following equation:

$$
x=y+r(y) t \quad \text { for }-T<t<T,
$$

where $r(y)$ can take on different values as a function of age, $y$, subject to certain restrictions (see section A-2.3); and $T$ is the duration of the shift both forward and backward from $t=0$. Note that $\frac{d x}{d t}=r(y)$ for all combinations of $x$ and $t$ along this line. In other words,

$$
\Phi_{p}(x, t)=\Phi(y) \quad \text { or } \quad \ell_{p}(x, t)=\ell(y)
$$

where $y=x-r(y) t$. Note that $\frac{d y}{d x}=1 /\left(1+r^{\prime}(y) t\right)$, where $r^{\prime}(y)=\frac{d}{d y} r(y)$. 
Thus, $\Phi(y)$ and $\ell(y)$ depict the baseline mortality distribution and survival probabilities for the linear shift model. They are identical to the corresponding period mortality functions associated with time $t=0$ for the model population (i.e., $\Phi_{p}(y, 0)=\Phi(y)$ and $\left.\ell_{p}(y, 0)=\ell(y)\right)$. Differentiating $\Phi_{p}(x, t)$ with respect to age, we obtain the period distribution of deaths by age:

$$
\phi_{p}(x, t)=\frac{d}{d x} \Phi_{p}(x, t)=\frac{d}{d y} \Phi(y) \cdot \frac{d y}{d x}=\frac{\phi(y)}{1+r^{\prime}(y) t} .
$$

Then, using the change of variable from $x$ to $y$ given above, we may calculate period life expectancy at birth as follows:

$$
\begin{aligned}
e_{0}^{p}(t) & =\int_{0}^{\infty} x \phi_{p}(x, t) d x \\
& =\int_{0}^{\infty}(y+r(y) t) \phi(y) d y \\
& =\int_{0}^{\infty} y \phi(y) d y+t \int_{0}^{\infty} r(y) \phi(y) d y \\
& =e_{0}+\bar{r} t,
\end{aligned}
$$

where $e_{0}=\int_{0}^{\infty} y \phi(y) d y$ and $\bar{r}=\int_{0}^{\infty} r(y) \phi(y) d y$.

Furthermore, the death rate at age $x$ and time $t$ is given by:

$$
\mu(x, t)=\mu_{p}(x, t)=\frac{\phi_{p}(x, t)}{\ell_{p}(x, t)}=\frac{\mu(y)}{1+r^{\prime}(y) t},
$$

where $\mu(y)=\phi(y) / \ell(y)=-\frac{d}{d y} \ell(y) / \ell(y)$ is the death rate at age $y$ implied by the baseline mortality distribution. For the cohort born at time $\tau$, the death rate at age $x$ is as follows:

$$
\mu_{c}(x, \tau)=\mu(x, \tau+x)=\frac{\mu(z)}{1+r^{\prime}(z)(\tau+x)},
$$

where $z=x-r(z)(\tau+x)$. Note that $\frac{d z}{d x}=(1-r(z)) /\left(1+r^{\prime}(z)(\tau+x)\right)$. Also note that $x=z+s(z)(\tau+z)$, where $s(z)=r(z) /(1-r(z))$. 
Now, using the change of variable from $x$ to $z$ given above, we can compute the cumulative death rate for the cohort born at time $\tau$ as follows:

$$
H_{c}(a, \tau)=\int_{0}^{a} \mu_{c}(x, \tau) d x=\int_{0}^{a} \frac{\mu(z)}{1+r^{\prime}(z)(\tau+x)} d x=\int_{0}^{b} \mu^{*}(z) d z=H^{*}(b)
$$

where $b=a-r(b)(\tau+a)$; and $\mu^{*}(z)=\mu(z) /(1-r(z))$. Based on this formula, it is simple to compute the probability of survival to age $x$ for the cohort born at time $\tau$ :

$$
\ell_{c}(x, \tau)=e^{-H_{c}(x, \tau)}=e^{-H^{*}(z)}=e^{-\int_{0}^{z} \mu^{*}(a) d a}=\ell^{*}(z)
$$

Therefore, the cohort distribution of deaths by age is as follows:

$$
\phi_{c}(x, \tau)=\ell_{c}(x, \tau) \mu_{c}(x, \tau)=\frac{\ell^{*}(z) \mu(z)}{1+r^{\prime}(z)(\tau+x)}=\frac{(1-r(z)) \phi^{*}(z)}{1+r^{\prime}(z)(\tau+x)}
$$

where $\phi^{*}(z)=\ell^{*}(z) \mu^{*}(z)$. Then, using the same change of variable from $x$ to $z$, we can calculate cohort life expectancy at birth as follows:

$$
\begin{aligned}
e_{0}^{c}(\tau) & =\int_{0}^{\infty} x \phi_{c}(x, \tau) d x \\
& =\int_{0}^{\infty}(z+s(z)(\tau+z)) \phi^{*}(z) d z \\
& =\int_{0}^{\infty} z \phi^{*}(z) d z+\tau \int_{0}^{\infty} s(z) \phi^{*}(z) d z+\int_{0}^{\infty} z s(z) \phi^{*}(z) d z \\
& =e_{0}^{*}+\bar{s} \tau+\int_{0}^{\infty} z s(z) \phi^{*}(z) d z,
\end{aligned}
$$

where $e_{0}^{*}=\int_{0}^{\infty} z \phi^{*}(z) d z$ and $\bar{s}=\int_{0}^{\infty} s(z) \phi^{*}(z) d z$

\section{A-2.2 Equivalence of period- and cohort-based models}

The linear shift model described in the main text and in the previous sub-section is specified in terms of period mortality. In other words, mortality change for the model population is described as a shift in the distribution of age at death in a series of period 
life tables. Thus, by design the period percentile slope at age $x$ in year $t, r_{p}(x, t)$, equals $r(y)$, where $y=x-r(y) t$. We can confirm this relationship by differentiating the period survival probability, $\ell_{p}(x, t)$, with respect to time $t$ :

$$
\frac{d}{d t} \ell_{p}(x, t)=\frac{d}{d y} \ell(y) \frac{d y}{d t}=\frac{\phi(y)}{1+r^{\prime}(y) t} r(y)=\phi_{p}(x, t) r(y),
$$

since $\frac{d}{d y} \ell(y)=-\phi(y)$ and $\frac{d y}{d t}=-r(y) /\left(1+r^{\prime}(y) t\right)$. Then, by the same logic used earlier to compute cohort percentile slopes (see section A-1 above), it follows that:

$$
r_{p}(x, t)=\frac{\frac{d}{d t} \ell_{p}(x, t)}{\phi_{p}(x, t)}=r(y) .
$$

Note that this result merely reflects the core assumption of the model.

On the other hand, values of cohort percentile slopes in this model are a consequence of the assumptions and must be derived. As before, the change of variable used for computing cohort mortality is defined by the equation, $z=x-r(z)(\tau+x)$. Differentiating the cohort survival probability, $\ell_{c}(x, t)$, with respect to time $\tau$, we obtain:

$$
\frac{d}{d \tau} \ell_{c}(x, \tau)=\frac{d}{d z} \ell^{*}(z) \frac{d z}{d \tau}=\frac{(1-r(z)) \phi^{*}(z)}{1+r^{\prime}(z)(\tau+x)} \frac{r(z)}{1-r(z)}=\phi_{c}(x, \tau) s(z)
$$

since $\frac{d}{d z} \ell^{*}(z)=-\phi^{*}(z)$ and $\frac{d z}{d \tau}=-r(z) /\left(1+r^{\prime}(z)(\tau+x)\right)$. It follows that:

$$
s_{c}(x, \tau)=\frac{\frac{d}{d \tau} \ell_{c}(x, \tau)}{\phi_{c}(x, \tau)}=s(z)
$$

Thus, a key property of the linear shift model is the close relationship between the period and cohort percentile slopes:

$$
s_{c}(x, \tau)=\frac{r_{p}(x, t)}{1-r_{p}(x, t)} \quad \text { and } \quad r_{p}(x, t)=\frac{s_{c}(x, \tau)}{1+s_{c}(x, \tau)} .
$$


Turning the derivation around, we begin by specifying that the percentiles of successive cohort distributions of age at death are constant along a line defined by

$$
x=z+s(z)(\tau+z) \text { for }-T<t<T .
$$

Note that $\frac{d x}{d \tau}=s(z)$ for all combinations of $x$ and $\tau$ along this line. In other words, the linear shift model is now based on the core assumption that

$$
\Phi_{c}(x, \tau)=\Phi^{*}(z) \text { or } \quad \ell_{c}(x, \tau)=\ell^{*}(z)
$$

where $z=x-s(z)(\tau+z)$. Since $\tau=-x$ at time $t=0$, this assumption implies that

$$
\Phi_{c}(z,-z)=\Phi^{*}(z) \quad \text { or } \quad \ell_{c}(z,-z)=\ell^{*}(z) \text {. }
$$

Therefore, the functions, $\Phi^{*}(z)$ and $\ell^{*}(z)$, again depict the cross-sectional cohort mortality distribution at time $t=0$ for the model population. From equation 64 we obtain $\frac{d z}{d x}=1 /\left(1+s(z)+s^{\prime}(z)(\tau+z)\right)$.

Note that both the change of variable implied by equation 64 , and the derivative of $z$ with respect to $x$ that follows from this equation, are equivalent to the forms given earlier as part of the original derivation of the linear shift model. To demonstrate the equivalence of the change of variable itself, add $\tau$ to both sides of equation 61 and rearrange the equation to obtain $\tau+x=(1+s(z))(\tau+z)$. Since $1+s(z)=(1-r(z))^{-1}$, it follows that $\tau+z=(1-r(z))(\tau+x)$, and thus $z=x-r(z)(\tau+x)$. Equivalence of the two forms of the derivative, $\frac{d z}{d x}$, follows from the additional observation that $s^{\prime}(z) /(1+s(z))=r^{\prime}(z) /(1-r(z))$. In summary:

$$
z=x-s(z)(\tau+z)=x-r(z)(\tau+x),
$$

and

$$
\frac{d z}{d x}=\frac{1}{1+s(z)+s^{\prime}(z)(\tau+z)}=\frac{1-r(z)}{1+r^{\prime}(z)(\tau+x)} .
$$


Differentiating $\Phi_{c}(x, \tau)$ with respect to age, the cohort distribution of deaths by age is as follows:

$$
\phi_{c}(x, \tau)=\frac{d}{d x} \Phi_{c}(x, t)=\frac{d}{d z} \Phi^{*}(z) \cdot \frac{d z}{d x}=\frac{\phi^{*}(z)}{1+s(z)+s^{\prime}(z)(\tau+z)} .
$$

Dividing through by $\ell_{c}(x, \tau)=\ell^{*}(z)$, we obtain the death rate at age $x$ for the cohort born at time $\tau$ :

$$
\mu_{c}(x, \tau)=\frac{\mu^{*}(z)}{1+s(z)+s^{\prime}(z)(\tau+z)}=\frac{(1-r(z)) \mu^{*}(z)}{1+r^{\prime}(z)(\tau+x)} .
$$

Finally, the death rate at age $x$ and time $t$ is as follows:

$$
\mu(x, t)=\mu_{p}(x, t)=\mu_{c}(x, t-x)=\frac{\mu(y)}{1+r^{\prime}(y) t},
$$

where $y=x-r(y) t$ and $\mu(y)=(1-r(y)) \mu^{*}(y)$.

Therefore, the surface of death rates over age and time, $\mu(x, t)$, has the same form, and thus the relationship between all period and cohort mortality functions is the same, whether a linear shift model is specified in terms of period or cohort mortality. However, as noted in the main text, this equivalence pertains only to periods and cohorts whose entire life experience takes place within the shift. In other words, discontinuities at the start and end of the shift have different forms for period-based and cohort-based models. Since the purpose of this analysis is to understand the relationship between period and cohort mortality under conditions of a sustained change over time, such patterns are not considered here.

\section{A-2.3 Restrictions on values of percentile slopes}

Although the percentile slopes of the linear shift model, $r(y)$ and $s(y)$, typically take on rather small values (often in a range between -1 and +1 ), there do not appear to be strict upper or lower limits on their possible values except at age zero (see below). However, a general restriction is necessary concerning the rate of change in either $r(y)$ or $s(y)$ as a function of age. The discussion here is preliminary and does not consider 
all possible cases. In particular, it considers only the necessary restrictions on $r(y)$ or $s(y)$ individually, without attempting to resolve the problem of how these two sets of restrictions are interrelated. Thus, the discussion is developed here in terms of $r(y)$ alone, without considering the case of $s(y)$ except to note that it should be subject simultaneously to a similar set of restrictions.

The first issue is how to avoid a cross-over of percentile trends over the course of the linear shift. Suppose that $a$ and $b$ are two ages such that $0 \leq a<b<\infty$. Consider the trends in the (period) percentiles associated with these two ages at time 0 . To avoid a cross-over in these two percentile trends, the following equation must be true for all $t$ in the range of $(-T, T)$ :

$$
a+r(a) t \leq b+r(b) t
$$

The following condition is both necessary and sufficient to ensure that the relationship in equation 72 remains true throughout the shift period:

$$
\left|r^{\prime}(y)\right| \leq \frac{1}{T} \text { for } 0 \leq y<\infty
$$

To show that equation 73 is necessary, start by rearranging equation 72 as follows:

$$
-t[r(b)-r(a)] \leq b-a
$$

Dividing both sides of equation 74 by $-t$ and $b-a$, and considering separately the two cases when $t$ is positive and negative, yields the following:

$$
\left|\frac{r(b)-r(a)}{b-a}\right| \leq\left|\frac{1}{t}\right| \text { for }-T<t<T \quad(\text { except } t=0)
$$

Since equation 75 must hold true throughout the shift period, it follows that

$$
\left|\frac{r(b)-r(a)}{b-a}\right| \leq \frac{1}{T}
$$

And since equation 76 must be true for all values of $a$ and $b$, including the case where these two ages are arbitrarily close together, equation 73 is a necessary condition. 
To show that equation 73 is sufficient, begin by noting the following relationship:

$$
r(b)-r(a)=\int_{a}^{b} r^{\prime}(y) d y \leq \int_{a}^{b}\left|r^{\prime}(y)\right| d y \leq \int_{a}^{b} \frac{1}{T} d y=\frac{b-a}{T} .
$$

The second inequality in this equation is implied by equation 73. Similarly,

$$
r(b)-r(a)=\int_{a}^{b} r^{\prime}(y) d y \geq-\int_{a}^{b}\left|r^{\prime}(y)\right| d y \geq-\int_{a}^{b} \frac{1}{T} d y=-\frac{b-a}{T} .
$$

Equations 77 and 78 together imply equation 76, which ensures the original restriction on $r(y)$ as expressed in equation 72 .

Thus, an important restriction on the possible values of percentile slopes in a linear shift model is that they should not change too quickly as a function of age. This conclusion appears closely related to the empirical observation that projected values of life expectancy based on the linear shift model are more plausible when percentile slopes are computed from smoothed mortality surfaces (see end of section 5.4).

Another important restriction concerns the value of the percentile slope at age 0 . In order to avoid negative ages at death for a shift from time $-T$ to $T$, it is necessary to require that

$$
r(0)=0 \quad .
$$

However, two alternative approaches are also worth considering. First, for a shift from time 0 to $T$, a weaker restriction is sufficient:

$$
r(0) \geq 0
$$

Or second, keeping the longer shift interval, $(-T, T)$, define an age, $a_{0}$, such that mortality rates equal zero below that age at time $t=0$. To avoid negative ages at death in this situation requires the following restriction:

$$
\left|r\left(a_{0}\right)\right| \leq \frac{a_{0}}{T}
$$


Although negative ages at death should be treated as strictly impossible, a complete absence of mortality within some age range might be considered possible though highly unlikely. A useful special case is the parallel linear shift model, in which $r(y)=r$ is constant over the full age range. This model has been applied by Bongaarts and Feeney $(2002,2003)$, who assume that mortality is zero below age 30 at the start of a parallel linear shift. Given the very low levels of mortality at younger ages observed in many modern populations, the inexactness of such an assumption may be outweighed in many situations by the advantages of working with a simpler model. 